\title{
THE FUNDAMENTAL THEOREMS OF ELEMENTARY GEOMETRY. AN AXIOMATIC ANALYSIS
}

\author{
BY \\ REINHOLD BAER
}

Introduction. The present investigation is concerned with an axiomatic analysis of the four fundamental theorems of Euclidean geometry which assert that each of the following triplets of lines connected with a triangle is copunctual: the medians, the altitudes, the perpendicular bisectors, and the bisectors of the angles. The general framework for our discussion will be provided by an affine plane (which is obtained from a projective plane by deleting a line and its points). But in order to enunciate these theorems we have to add to the concepts provided by affine geometry two further relations: the relation of the midpoint and that of orthogonality.

There exists one important difference between these two relations. If one subjects the midpoint relation to some obvious and formal restrictions, then there exists at most one such relation. But there exist always different orthogonality relations, since affine transformations transform one such relation into a different one; and even if one considers such orthogonality relations as not essentially different and considers only orthogonality relations meeting quite a fair amount of requirements, then uniqueness will be an exceptional case.

The existence of a midpoint relation is equivalent to the closure of certain configurations, to the existence of sufficiently many reflections in points, and to the following algebraic criterion: the plane under consideration is the plane over a right distributive Cartesian number system of characteristic different from 2 (using a concept introduced in an earlier paper $\left(^{1}\right)$ ). If the characteristic is 3 , then the medians of a triangle are parallel, otherwise they are copunctual.

Given a midpoint relation and an orthogonality relation meeting the obvious and formal requirements, then the theorem of the altitudes and that of the perpendicular bisectors of a triangle are equivalent; and they are both equivalent to the fact that the plane under consideration is the plane over an ordinary commutative field of characteristic different from 2 and that orthogonality may be defined in terms of a quadratic form $y^{2}-c x^{2}, c \neq 0$. The relation between these theorems and the theorem of the bisectors of the angles is not as clearcut. For, the latter theorem is a consequence of the former ones; but the converse can be obtained only by adding two further statements concerning the existence of bisectors of angles, which does not seem to be assured by

Presented to the Society, April 29, 1944 ; received by the editors January 5, 1944.

(1) Baer [1, p. 145]; numbers in brackets refer to the Bibliography at the end of this paper. 
the assertion of their copunctuality (this contention being void, if there do not exist any bisectors of the angles).

There exist elementary definitions of congruence in terms of orthogonality, and vice versa. It is of interest to note that the congruence relation thus obtained is transitive if, and only if, the theorem of the copunctuality of the altitudes in a triangle is true. On the other hand one has to add to the formal properties of congruence the fact that the points of equal distance from two different points form a line in order to assure that a given congruence relation may be derived from and leads to a satisfactory orthogonality relation.

The problem we are discussing has been touched upon in investigations concerning absolute geometry $\left({ }^{2}\right)$. But as we restrict ourselves to Euclidean geometry, our results appear accordingly more precise. Furthermore we do not make any hypotheses concerning order or betweenness, nor do we assume the existence of points of a given distance on given lines nor the existence of reflections in lines interchanging two given lines. As a matter of fact these latter hypotheses, usually adopted in treatments of absolute geometry $\left({ }^{2}\right)$, are in general not satisfied.

\section{Chapter I: Midpoints, Reflections ANd COORdinates}

I.1. The affine plane. For our purposes it will be most convenient to obtain the affine plane of our considerations by distinguishing a line in a projective plane. Thus let $E$ be a projective plane consisting of points and lines which are connected by the relation $P<p$, which reads either "the point $P$ is on the line $p$ " or "the line $p$ passes through the point $P$." These concepts are subject to the following postulates.

A. If $P$ and $Q$ are two different points, then there exists one and only one line $P+Q=Q+P$ which passes through both points.

B. If $p$ and $q$ are two different lines, then there exists one and only one point $p q=q p$ which is on both lines.

$A^{\prime}$. Every line passes through three different points.

$B^{\prime}$. Every point is on three different lines.

To descend from projective geometry to affine geometry we distinguish one line in $E$ which we term the ideal line $i$ or the line at infinity, and the points on $i$ are termed ideal points or points at infinity. Lines different from $i$ are ordinary lines; and lines are termed parallel, in symbols $p \| q$, if $p q$ is a point on $i$, an ideal point, or if they are equal.

From a puristic point of view it would have been better to introduce parallelism as a fundamental concept and to work without the use of ideal points. This would have been very easy. But for our present purposes this part of the axiomatic system is not important; and thus the shortest way is the best and most convenient.

(2) See in particular the work of F. Bachmann, O. Bottema, J. Hjelmslev, F. Schur, G. Thomsen and H. Toepken. 
I.2. The postulates for midpoints. If $P$ and $Q$ are two different points in the affine plane, then their sum $P+Q$ is a well determined line in this plane. We shall now introduce a ternary relation written as $P \cdot Q=R$ and to be read either as " $R$ bisects (the segment) $P Q$ " or " $R$ is the midpoint of $P Q$ "; and this relation shall be subject to the following postulates.

I. If $P \cdot Q=R$, then $P, Q, R$ are three different points on the same line.

II. $P \cdot Q=R$ implies $Q \cdot P=R$.

If $P$ and $Q$ are two different points, then there exists:

III.E. at least one point $R$ such that $P \cdot Q=R$.

III.U. at most one point $R$ such that $P \cdot Q=R$.

IV.E. at least one point $R$ such that $P \cdot R=Q$.

IV.U. at most one point $R$ such that $P \cdot R=Q$.

The purely formal nature of these postulates will become still clearer if we in troduce the convenient rules: $P+P=P=P \cdot P$, and restate $I$ in the form: $P+Q=Q+P \cdot Q=P \cdot Q+P$.

The final postulate is less formal. It states the invariance of the midpoint relation under parallel projections.

V. If $P \cdot Q=R$, if $P^{\prime}, Q^{\prime}, R^{\prime}$ are collinear points, and if there exist three different, but parallel, lines $p, q, r$ such that $P, P^{\prime}$ are on $p, Q, Q^{\prime}$ on $q$, and $R, R^{\prime}$ on $r$, then $P^{\prime} \cdot Q^{\prime}=R^{\prime}$.

Note that the points $X$ and $X^{\prime}$ on the line $x$ may be equal.

These postulates are redundant. We shall have to point out some of these redundancies. Furthermore there exist a number of equivalent properties which will prove important later on.

THEOREM 1. If the postulates I, II, and V are satisfied by the midpoint relation $X \cdot Y=Z$, then

(a) III.E and IV.E are equivalent properties, and

(b) III.U and IV.U are equivalent properties of this relation.

Proof. To any pair of different points $K$ and $L$ there exists a point $M$, not on the line $K+L$. If III.E is valid, then there exists a point $A$ such that $A=K \cdot M$. There exists one and only one line $m$ through $M$ which is parallel to $A+L$; and we infer from V that $L=[m(K+L)] \cdot K$, showing that IV.E is a consequence of III.E. If conversely IV.E holds true, then there exists a point $H$ on $K+M$ such that $M=K \cdot H$. There exists one and only one line $h$ through $M$ which is parallel to $H+L$; and we deduce $h(K+L)=K \cdot L$ from V, proving the equivalence of III.E and IV.E.

Assume next the validity of III.U. If $N^{\prime}, N^{\prime \prime}$ are points such that $L=K \cdot N^{\prime}$ and $L=K \cdot N^{\prime \prime}$, then denote by $n^{\prime}, n^{\prime \prime}$ the uniquely determined lines through $L$ which are parallel to $M+N^{\prime}$ and $M+N^{\prime \prime}$ respectively. We infer from $\mathrm{V}$ that $n^{\prime}(K+M)=K \cdot M$ and that $n^{\prime \prime}(K+M)=K \cdot M$. Now the equality of $n^{\prime}$ and $n^{\prime \prime}$ is a consequence of III.U; and this equality implies $N^{\prime}=N^{\prime \prime}$, showing that IV.U is a consequence of III.U. If conversely IV.U is 
satisfied, then let $B^{\prime}, B^{\prime \prime}$ be points satisfying $B^{\prime}=K \cdot L$ and $B^{\prime \prime}=K \cdot L$. There exist uniquely determined lines $b^{\prime}, b^{\prime \prime}$ through $L$ which are parallel to $M+B^{\prime}$ and $M+B^{\prime \prime}$ respectively. We deduce from $\mathrm{V}$ that $M=\left[b^{\prime}(K+M)\right] \cdot K$ and that $M=\left[b^{\prime \prime}(K+M)\right] \cdot K$. Hence $b^{\prime}=b^{\prime \prime}$ and therefore $B^{\prime}=B^{\prime \prime}$ are consequences of IV.U, proving the equivalence of III.U and IV.U.

TheOREM 2. If postulates I to $\mathrm{V}$ are satisfied by the midpoint relation $X \cdot Y=Z$, then the following three properties of the three different collinear points $P, Q, R$ imply each other.

(a) $P \cdot Q=R$.

(b) If $S$ and $T$ are points not on the line $P+Q$, if $R, S, T$ are three different collinear points, then $P+S \| Q+T$ implies $P+T \| Q+S$.

(c) There exist points $S, T$ not on $P+Q$ such that $R, S, T$ are three different collinear points and such that both $P+S \| Q+T$ and $P+T \| Q+S$.

Proof. If $P \cdot Q=R$, and if $R, S, T$ are three different collinear points such that $S+T \neq P+Q$ and such that $P+S \| Q+T$, then we deduce $S \cdot T=R$ from V. Denote by $q$ the uniquely determined line through $S$ parallel to $P+T$. Then $q$ is not parallel to $P+Q$ and meets this line in a point $Q^{\prime}$. It follows from $\mathrm{V}$ and $S \cdot T=R$ that $P \cdot Q^{\prime}=R$; and $Q=Q^{\prime}$ is a consequence of IV.U, proving that $Q+S=q \| P+T$. Thus (b) is a consequence of (a).

If (b) is satisfied by the three collinear points $P, Q, R$, then there exists a point $S$ not on $P+Q$. There exists one and only one line $q$ through $Q$ which is parallel to $P+S$. This line $q$ is not parallel to $R+S$ so that these two lines meet in a well determined point $T$. We deduce from (b) that $P+T$ and $Q+S$ are parallel, proving that (c) is a consequence of (b).

Assume finally the validity of (c). Then there exist points $S, T$ such that the points $P, Q, R, S, T$ meet the requirements of (b). We infer from III.E the existence of a point $R^{\prime}$ such that $P, Q, R^{\prime}$ are three different collinear points satisfying $P \cdot Q=R^{\prime}$. The lines $S+R^{\prime}$ and $Q+T$ are not parallel so they have one and only one point $T^{\prime}$ in common. Then $S, R^{\prime}, T^{\prime}$ are three different collinear points such that $P+S \| Q+T^{\prime}$ and $P \cdot Q=R^{\prime}$. We have already shown that (b) is a consequence of (a) and hence it follows that $P+T^{\prime} \| Q+S$. Since $Q+S$ and $P+T$ are parallel lines by hypothesis, we deduce that $T=T^{\prime}$ and this is readily seen to imply $R=R^{\prime}$. Thus (a) is a consequence of (c).

We introduce now an operation as follows.

Definition. If $P, Q, R$ are three different collinear points satisfying condition (c) of Theorem 2, then $P \circ Q=R$.

It is an immediate consequence of Theorem 2 that the operations $P \circ Q$ and $P \cdot Q$ coincide if the latter is a midpoint relation meeting the requirements $I$ to $V$. Thus there exists in a given affine plane at most one midpoint relation meeting the requirements $I$ to $V$. It is our object to find conditions 
assuring that the postulates $\mathrm{I}$ to $\mathrm{V}$ are satisfied by the relation $P \circ Q=R$. We note that I and II are always satisfied.

TheOREM 3. Postulate III is satisfied by the relation $X \circ Y=Z$ if, and only if, the plane has the following property.

(iii) If $A$ and $A^{\prime}$ are two different points, if $a, b, u, v$ are lines through $A$, different from $A+A^{\prime}$, if $a \neq b$ and $u \neq v$, and if the lines $a^{\prime}, b^{\prime}, u^{\prime}, v^{\prime}$ through $A^{\prime}$ are parallel to $a, b, u, v$ respectively, then the lines $A+A^{\prime}, a b^{\prime}+a^{\prime} b$ and $u v^{\prime}+u^{\prime} v$ have one point in common.

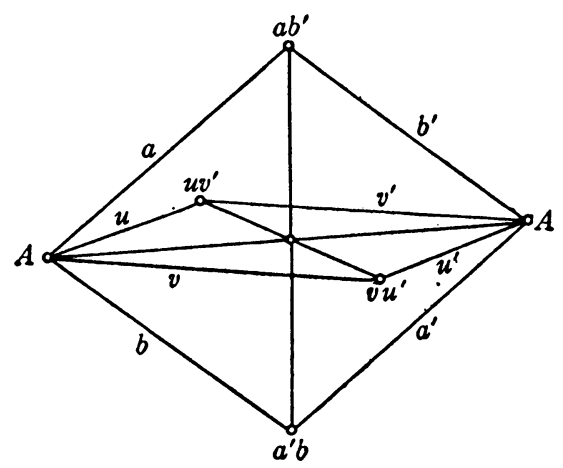

Condition (iii)

FIG. 1

Proof. If postulate III is satisfied by $X \circ Y=Z$, then the common point of the three lines mentioned at the end of (iii) is the uniquely determined point $A \circ A^{\prime}$. For there exist points $S, T$, not on $A+A^{\prime}$, such that $A \circ A^{\prime}$ is both on $A+A^{\prime}$ and on $S+T$ and such that $A+S \| A^{\prime}+T$ and $A+T \| A^{\prime}+S$. Hence it follows from the definition that $A \circ A^{\prime}=R=S \circ T$. The line $a b^{\prime}+a^{\prime} b$ meets at least one of the two different and not parallel lines $A+A^{\prime}$ and $S+T$. But then it follows from III.U that it meets this line in $R$; and the same argument applies to $u v^{\prime}+u^{\prime} v$.

We assume now conversely that (iii) is satisfied by the plane. If $A$ and $A^{\prime}$ are two different points, then there exist lines $a, b$ through $A$ such that $a, b, A+A^{\prime}$ are three different lines. Denote by $a^{\prime}, b^{\prime}$ the lines through $A^{\prime}$ which are parallel to $a$ and $b$ respectively. Apply (iii) with $a=u$ and $b=v$. Then it follows from (iii) that $A+A^{\prime}$ and $a b^{\prime}+a^{\prime} b$ have a point in common, showing the existence of at least one point $A \circ A^{\prime}$, that is, the validity of III.E. The validity of III.U is a fairly immediate consequence of (iii) and the definition of $X \circ Y=Z$.

REMARK. In the proof of III.E we need only Fano's axiom:

The diagonals of a parallelogram are not parallel.

This is the special case $a=u, b=v$ of (iii). As a matter of fact for the proof of III.E the following still weaker postulate is sufficient: 
If $A . \neq A^{\prime}$, then there exists a parallelogram with vertices $A, A^{\prime}$ (not on the same edge) whose diagonals are not parallel.

THEOREM 4. If postulate III is satisfied by the relation $X \circ Y=Z$, then postulate $\mathrm{V}$ is equivalent to the following property.

(v) If no three of the four points $A, B, C, D$ are collinear, if $A+B \| D+C$, $A+D \| B+C$, if $F$ is a point on $A+D$ such that $B+D \| C+F$, then $A \circ F=D$.

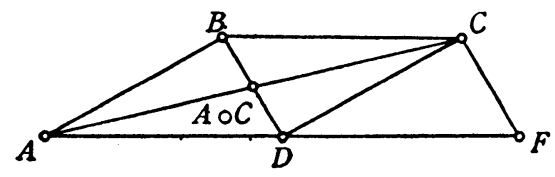

Condition (v)

Fig. 2

Proof. Suppose first that postulate $\mathrm{V}$ is satisfied by the relation $X \circ Y=Z$, and that the points $A, B, C, D, F$ meet all the requirements of (v). Then $A \circ C=(A+C)(B+D)$ by definition and III; and hence it follows from V that $A \circ F=D$.

Assume conversely that $(\mathrm{v})$ is satisfied by the plane. The proof of $\mathrm{V}$ will be effected in several steps.

1. Suppose that $P \circ Q^{\prime}=R^{\prime}$, that $P, R, Q$ are three different collinear points, and that-assuming that $R \neq R^{\prime}, Q \neq Q^{\prime}-R+R^{\prime} \| Q+Q^{\prime}$. There exists one and only one point $T$ such that $T+P \| R+Q^{\prime}$ and $T+Q^{\prime} \| P+R$. It is a consequence of Theorem 3 (iii) that $T, R^{\prime}, R$ are collinear and that therefore $T+R \| Q^{\prime}+Q$; and hence $P \circ Q=R$ is a consequence of (v).

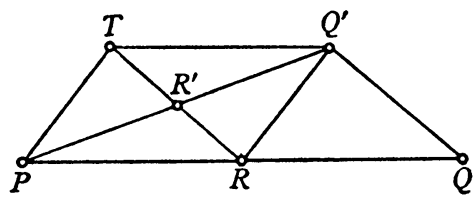

FIG. 3

2. Suppose that $P \circ Q=R$, that $P^{\prime}, Q^{\prime}, R^{\prime}$ are three different collinear points such that $P^{\prime}+Q^{\prime}$ and $P+Q$ are different, but parallel, lines and such that $P+P^{\prime}, Q+Q^{\prime}$, and $R+R^{\prime}$ are three parallel lines. Then it follows from the commutativity of the relation $X \circ Y=Z$ and from what we proved under 1 that

$$
P \circ R^{\prime}=\left(Q+Q^{\prime}\right)\left(P+R^{\prime}\right)
$$

and that therefore-for the same reasons- $R^{\prime} \circ P^{\prime}=Q^{\prime}$ and hence $P^{\prime} \circ R^{\prime}=Q^{\prime}$. 3. If $p, q, r$ are three different parallel lines, if $P, P^{\prime}$ are on $p, Q, Q^{\prime}$ on $q$, 
and $R, R^{\prime}$ on $r$, if $P \circ Q=R$, and if $P^{\prime}, Q^{\prime}, R^{\prime}$ are three collinear points, $Q \neq Q^{\prime}$, then let $w$ be the uniquely determined line through $Q$ which is parallel to $P^{\prime}+Q^{\prime}$. Then it follows from the result obtained under 1 that

$$
Q \circ w p=w r
$$

and it follows therefore from the result obtained under 2 that

$$
Q \circ P=R
$$

and that therefore

$$
P \circ Q=R
$$

completing the proof.

The results of this section may be summarized as follows.

In an affine plane there exists at most one midpoint relation satisfying the postulates I to V. The existence of such a relation is equivalent to the validity of the properties (iii) and (v) in the plane.

I.3. Reflections in points. The 1:1 correspondence $f$ between the points of the plane is termed a reflection in the point $R$, if

(a) $f^{2}=1, f \neq 1$,

(b) $R^{f}=R$,

(c) $P+Q \| P^{f}+Q^{f}$ for $P \neq Q$.

ThEOREM 1. If $f$ is a reflection in the point $R$, and if $P$ is a point different from $R$, then $R=P \circ P^{f}$ (in the notation of $\S I .2$ ).

Proof. Substituting $Q=R$ in condition (c) it follows that the lines $P+R$ and $P^{f}+R$ are parallel and therefore equal, showing the collinearity of $P, R, P^{f}$.

If all the points not on the line $P+R$ were fixed points of $f$, then it would follow from (c) that $f=1$, contradicting (a). Thus there exists a point $T$, not on $P+R$, which is not a fixed point under $f$. It follows as before that $T, R, T^{f}$ are three collinear points; and the line $T+R$ is clearly different from the line $P+R$. If $P$ were equal to $P^{f}$, then it would follow from (c) that the lines $P+T$ and $P+T^{f}$ were equal, implying the equality of $T$ and $T^{f}$, an impossibility which proves that $P$ is not a fixed point under $f$. From $f^{2}=1$ and (c) we deduce that $P+T \| P^{f}+T^{f}$ and that $P+T^{f} \| P^{f}+T$; and now $R=P \circ P^{f}$ is an immediate consequence of the definition of this operation.

TheOREM 2. If $P$ and $Q$ are different points, then there exists at most one reflection (in a suitable point) which interchanges $P$ and $Q$.

Proof. If $f$ is a reflection interchanging $P$ and $Q$, and if $T$ is a point not on the line $P+Q$, then $T^{f}$ is the uniquely determined point $X$ such that $T+P \| Q+X$ and $T+Q \| P+X$; and now it is clear how to finish the proof.

TheOREM 3. Postulates III and V (of §I.2) are satisfied by the operation 
$X \circ Y=Z$ if, and only if, there exists to every pair of different points a reflection interchanging them.

Proof. If Postulates III and V are satisfied by $X \circ Y=Z$, then it follows from I.2, Theorem 1, that all the postulates I to V are satisfied by this operation. If $P$ and $Q$ are two different points, then there exists one and only one point $R$ such that $P \circ Q=R$; and there exists to every point $X \neq R$ one and only one point $X^{*}$ such that $X \circ X^{*}=R$. It is clear that this transformation $f$ which maps $X$ upon $X^{*}$ interchanges $P$ and $Q$, is a $1: 1$ correspondence between the points of the plane, and satisfies conditions (a) and (b), provided we define $R=R^{f}$. If $X \neq R$, then $X, R, X^{f}$ are collinear. If $X, Y, R$ are three different points which are not collinear, then we infer from IV.U and the definition of the operation $S \circ T=L$ that the four points $X, Y, X^{*}, Y^{*}$ form a parallelogram whose diagonals meet in $R$, proving that $f$ is the desired reflection.

Assume conversely that there exists to every pair of different points a reflection interchanging them. Suppose now that $A$ and $A^{\prime}$ are two different points, that $a \neq b, u \neq v$ are lines through $A$ which are all of them different from the line $A+A^{\prime}$, and that the lines $a^{\prime}, b^{\prime}, u^{\prime}, v^{\prime}$ through $A^{\prime}$ are parallel to $a, b, u, v$ respectively. There exists a reflection $f$ in a point $R$ which interchanges $A$ and $A^{\prime}$. This reflection interchanges $a$ and $a^{\prime}, b$ and $b^{\prime}, u$ and $u^{\prime}$, $v$ and $v^{\prime}$. The lines $A+A^{\prime}, a b^{\prime}+a^{\prime} b$ and $u v^{\prime}+u^{\prime} v$ are therefore fixed lines under $f$ so that they all pass through $R$. This shows that the plane has property (iii) of I.2, Theorem 3, proving that the o-operation meets requirement

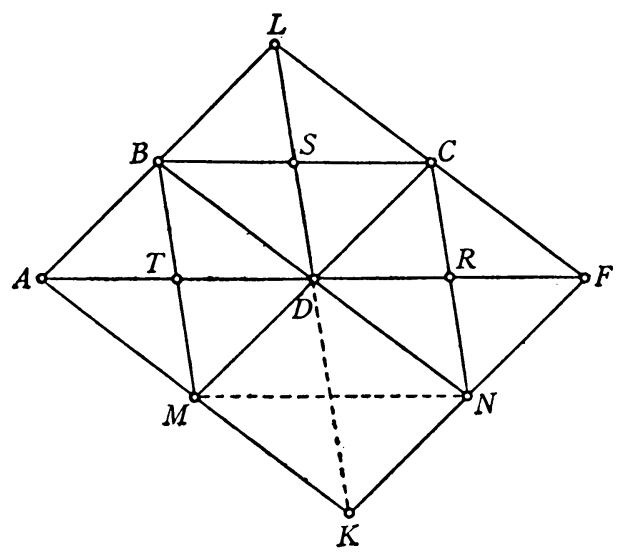

FIG. 4

III. Suppose next that no three of the four points $A, B, C, D$ are collinear, that $A+B\|C+D, A+D\| B+C$, and that $F$ is the point on $A+D$ such that $B+D \| C+F$. Denote by $L$ the common point of the lines $A+B$ and $C+F$, and by $K$ the uniquely determined point such that $A+K \| F+L$ and 
$F+K \| A+L$. Furthermore let $M=(A+K)(C+D), N=(F+K)(B+D)$, $R=(D+F)(C+N), S=(B+C)(D+L), T=(A+D)(B+M)$. There exists a reflection $f$ interchanging $C$ and $D$. This reflection interchanges $B$ and $F$ and hence $L$ and $N$, so that $C+N \| D+L$. There exists a reflection $g$ interchanging $B$ and $D$. This reflection interchanges $C$ and $A$ and therefore $L$ and $M$, proving $D+L \| M+B$. The transformation $f g$ maps $C$ upon $B$ and $N$ upon $M$; and it maps every line upon a parallel line, since both $f$ and $g$ have this latter property. Thus both $B+C$ and $M+N$ are fixed lines under $f g$. If these lines were not parallel, they would have a point $W$ in common and $W$ would be a fixed point for $f g$. Thus $W^{f}=W^{o}$ and it would follow from Theorem 2 that $f=g$. But this is impossible, since this would imply $B=C$. Thus we have shown $B+C \| M+N$. There exists finally a reflection $h$ interchanging $D$ and $M$. It interchanges $A$ and $N$ and therefore $B$ and $K$. Hence $B+M \| D+K$. Thus $D+L$ and $D+K$ have been shown to be parallel lines so that $D, L, K$ are collinear points. Hence we have shown that $A K F L$ is a parallelogram whose diagonals meet in $D$, proving $A \circ F=D$. Thus condition (v) of I.2, Theorem 4 , is satisfied in the plane, completing the proof.

COROLlARY. If there exists to every pair of different points in the plane a reflection interchanging them, then there exists to every pair of (equal or different) points one and only one reflection interchanging them.

Proof. Because of Theorem 2 it suffices to prove the existence of one and only one reflection in the point $R$ (for every $R$ ). It is a consequence of Theorem 3 that there exists to every point $X \neq R$ one and only one point $X^{*}$ satisfying $X \circ X^{*}=R$. Hence it follows from Theorem 1 that there exists at most one reflection in $R$. That mapping $R$ upon itself and the point $X \neq R$ upon the point $X^{*}$ satisfying $X \circ X^{*}=R$ is the desired reflection in $R$ may be deduced easily from Theorem 3 .

I.4. Translations and coordinates. The $1: 1$ correspondence $t$ between the points of the plane is termed a translation if

(a) $X+X^{t} \| Y+Y^{t}$ in case $X$ and $Y$ are not fixed points of $t$,

(b) $X+Y \| X^{t}+Y^{t}$ for $X \neq Y$.

If the translation $t$ is different from 1 , then the parallel lines occurring in condition (a) belong all to the same pencil of parallel lines, determine one and only one ideal point $J$; and we may say that $t$ is a translation in the direction $J$.

It is well known $\left(^{3}\right)$ that a translation possessing a fixed point is the identity. Thus two translations are equal if there exists one point which is mapped by both translations on the same point. The translations form a group $T$. This group is known to be commutative $\left({ }^{4}\right)$ if there exist translations in different directions.

(3) Proofs of this easily verified fact may be found, for example, in Artin [1] and Baer [1].

(4) For a proof cp. Artin [1]. 
LEMMA 1. The product of two reflections is a translation and the product of three reflections is a reflection.

Proof. If $f$ is a product of any number of reflections, then $f$ has the above property (b). If the point $P$ is not a fixed point under $f$, then the line $P+P^{f}$ is a fixed line under $f$, since it is mapped by $f$ upon a parallel line passing through $P^{\prime}$.

If $r$ and $s$ are reflections, then it follows from I.3, Theorem 2, that the existence of one fixed point of $r s$ implies $r=s$ and therefore $r s=1$. But if $r s$ has no fixed point, then it follows from the result of the first paragraph of the proof that all the lines $P+P^{r s}$ are parallel, showing that $r s$ is a translation.

If $t$ is a translation and $r$ a reflection in a point $R$, and if $P$ is some point, then $P+P^{t} \| P^{t r}+\left(P^{t r}\right)^{t}$ and $P+P^{t r} \| P^{t}+P^{t r t}$. This implies $P^{r}=P^{t r t}$ or $r=t r t$, since $r^{2}=1$; and now it is readily verified that $t r$ is a reflection. Since we have shown $t^{-1}=r^{-1} t r$, we have verified the following fact.

COROLlaRY 1. If there exist reflections, if $R$ is the group generated by the reflections, then $T$ is a subgroup of index 2 in $R$, and $T$ is abelian.

An almost immediate consequence of Lemma 1 and of I.3, Corollary, is the following important fact.

COROLLARY 2. There exists to every pair of different points a reflection interchanging them if, and only if, there exist reflections and the group of translations is (simply) transitive.

In the following we shall need the concept of Cartesian number system $\left(^{(5)}\right.$. This is a set $F$ of elements with double composition: addition $m+n$ and multiplication $m n$, subject to the following rules.

(1) $F$ is a group with regard to addition.

(2) The product $m n$ of the elements $m$ and $n$ (in this order) is a uniquely determined element in $F$.

(3) $0 m=m 0=0$ for every $m$ in $F$.

(4) There exists an element $1 \neq 0$ in $F$ satisfying $1 m=m, m(-1)=-m$ for every $m$ in $F$.

(5) If $r, s, t$ are elements in $F$ such that $r \neq s$, then there exists one and only one element $x$ and one and only one element $y$ in $F$ such that

$$
-x s+x r=t, \quad s y-r y=t .
$$

We shall make use of the following theorem which has been proved elsewhere $\left(^{(6)}\right.$.

Lemma 2. If $R, S, T$ are three noncollinear points in the plane, then the following properties imply each other.

(5) Baer [1, p. 145].

(6) Baer [1, Theorem 4.1, p. 146]. 
(c) If $X$ is a point on the line $R+S$, then there exists a translation mapping $R$ upon $X$.

(d) There exists a Cartesian number system $F$ and a 1:1 correspondence $f$ mapping the points of the plane upon the set of all the pairs $(x, y)$ of numbers in $F$, meeting the following requirements.

(d') $R^{f}=(0,0), S^{f}=(0,1), T^{f}=(1,0)$.

$\left(\mathrm{d}^{\prime \prime}\right)$ The lines parallel to $R+S$ are mapped by $f$ upon the sets $x=$ const. and the other lines are mapped by $f$ upon the loci satisfying $y=x r+s$.

Condition $\left(\mathrm{d}^{\prime \prime}\right)$ contains a description of an affine plane and we shall refer to this affine plane as the plane over $F$. If there exists in the plane over $F$ a translation mapping the point $(0,0)$ upon the point $(a, b)$, then this translation may be described by the following transformation formulas: $x^{\prime}=x+a$, $y^{\prime}=y+b$.

We need two more facts.

Lemma 3( $\left.{ }^{7}\right)$. The group of translations of the plane over $F$ is transitive if, and only if,

$$
(u+v) w=u w+v w \text { for } u, v, w \text { in } F .
$$

Proof. If (6) is satisfied by $F$, then it is readily seen that the formulas $x^{\prime}=x+a, y^{\prime}=y+b$ define a translation of the plane over $F$, showing that (6) implies the transitivity of the group of translations.

Suppose conversely that the group of translations be transitive. Then this group is commutative, as has been remarked before. If $u$ is any element in $F$, then there exists a translation mapping $(0,0)$ upon $(-u, 0)$; and one verifies that the transformation formulas of this translation are $x^{\prime}=x-u, y^{\prime}=y$. From this fact and the commutativity of the group of translations we deduce the commutativity of addition in $F$. This translation maps the line $y=x r$ upon the line $y=(x+u) r$ which has to be of the form $y=x r+s$. Substituting $x=0$ we find $s=u r$; and thus it follows now that

$$
(x+u) r=x r+u r
$$

as was to be shown.

Lemma 4. If the Cartesian number system $F$ satisfies the distributive law (6), then the following properties imply each other.

$$
1+1 \neq 0 \text {. }
$$

(iii') The diagonals of a parallelogram are not parallel in the plane over $F$.

(e) There exists a reflection in the plane over $F$.

Proof. If $1+1 \neq 0$, then $1 \neq-1$. It is readily verified that then a reflection

${ }^{(7)}$ This lemma may be used for a simple solution of certain problems proposed by R. Moufang [1]. 
in the origin is defined by the formulas $x^{\prime}=-x, y^{\prime}=-y$. Conversely if there exists a reflection, then it follows from Lemmas 2 and 3 and from Corollary 2 and I.3, Corollary, that there exists a reflection in the origin. This reflection $r$ maps the point $(1,0)$ upon a point $(r, 0)$ where $r$ is different from 0 and 1 . Thus $r$ maps the line $x=1$ upon the line $x=r$. Since the point $(1, c)$ is on the line $y=x c$ which is mapped upon itself by $r$, we find now $(1, c)^{r}=(r, r c)$. Thus in particular $(1,-1)$ is mapped upon $(r,-r)$ so that the line $y=-1$ is mapped upon the line $y=-r$. But the point $(1,-r)$ is mapped upon the point $(r, r(-r))=\left(r,-r^{2}\right)$ by (6); and the lines $y=-1$ and $y=-r$ are interchanged by $r$. Hence $-r^{2}=-1$, proving that $1 \neq r=-1$; and (7) has been shown to be a consequence of (e).

If (iii') holds true, then we consider the parallelogram $(0,0),(0,1),(1,1)$, $(1,0)$. Its diagonals are the lines $y=x \cdot 1$ and $y=-x+1$. These are not parallel, proving $1 \neq-1$ so that (iii') implies (7).

Suppose finally that (7) be satisfied, and the points $A, B, C, D$ form a parallelogram. It is a consequence of Lemma 2 that there exists a Cartesian number system $\bar{F}$ such that our plane may be considered a plane over $\bar{F}$ and such that in this system of coordinates the points $A, B, C, D$ are the points $(0,0),(0,1),(1,1),(1,0)$ respectively. Since $(7)$ is satisfied by $F$, it follows from the result of the first paragraph of the proof that there exist reflections. Since (6) is satisfied by $F$, it follows from Lemma 3 that the group of translations is transitive. Hence it follows from Lemma 3 that (6) is satisfied by $\bar{F}$; and thus we may apply the result of the first paragraph of the proof on $\bar{F}$, showing that ( 7$)$ is satisfied by $\bar{F}$ too. That the diagonals of the parallelogram $A, B, C, D$ meet is now verified by direct computation, completing the proof.

The validity of the following fact is a direct consequence of Corollary 2 and the Lemmas 2, 3, 4 .

THEOREM. There exists to every pair of different points a reflection interchanging them if, and only if, the plane is the plane over a Cartesian number system which satisfies (6) and (7), that is, $F$ is right distributive and of characteristic not 2.

It should be noted that the characteristic of $F$ is an invariant of the plane. All the translations different from 1 have the same order (as elements in the group $T$ ); and this order is either 0 or a prime number, and is just the characteristic of $F$. Thus we shall term it the characteristic of the plane.

I.5. Similar triangles. The theorems of this section do not serve only as an illustration of the results obtained so far, but they will be needed in the future.

THEOREM 1. If there exists to every pair of different points a reflection interchanging them, if the three points $A, B, C$ are not collinear, and if $\left(^{8}\right) A^{\prime}=B \circ C$, $B^{\prime}=C \circ A$, then $A+B \| A^{\prime}+B^{\prime}$.

(8) For the definition of the operation $X \circ Y$, see I.2. 
Proof. It is a consequence of I.3, Theorem 3, that the reflection $a$ which interchanges $B$ and $C$ is the reflection in $A^{\prime}$, and that the reflection $b$ which interchanges $C$ and $A$ is the reflection in $B^{\prime}$. We deduce from I.4, Lemma 1 , that $a b$ is a translation. But $a b$ maps $B$ upon $A$ and leaves therefore the two lines $A+B$ and $A^{\prime}+B^{\prime}$ invariant. Since $A \neq B$, it follows that $a b \neq 1$, and that therefore all lines invariant under the translation $a b$ are parallel. Hence $A+B \| A^{\prime}+B^{\prime}$, as we desired to show.

THEOREM 2. If there exists to every pair of different points a reflection interchanging them, if the three points $A, B, C$ are not collinear, and if $A^{\prime}, B^{\prime}, C^{\prime}$ are three different points situated on the lines $B+C, C+A, A+B$ respectively, then the following two properties imply each other.

(a) $A^{\prime}=B \circ C, B^{\prime}=C \circ A, C^{\prime}=A \circ B$.

(b) $A+B\left\|A^{\prime}+B^{\prime}, B+C\right\| B^{\prime}+C^{\prime}, C+A \| C^{\prime}+A^{\prime}$.

REMARK. It is immediately seen that an affine plane has property $(v)$ of $\$ I .2$ if the properties (a) and (b) are equivalent.

Proof. That (a) implies (b) is an immediate consequence of Theorem 1. It is a consequence of I.3, Theorem 3, and of I.2, Theorem 4, that property I.2 (v) holds in the plane. This implies that (a) is a consequence of (b).

I.6. The medians of a triangle.

THEOREM. Assume that the affine plane admits of a midpoint relation satisfying postulates I to $\mathrm{V}$.

(a) If the characteristic of the plane is not 3 , then the medians of a triangle are copunctual.

(b) If the characteristic of the plane is 3 , then the three medians of a triangle are parallel.

Proof. Suppose that the three points $A, B, C$ are not collinear. Then there exists by I.4, Lemma 2, a representation of the plane by means of coordinates from a Cartesian number system $F$ such that $A=(0,0), B=(1,0)$, $C=(0,1)$. Denote by $A^{\prime}, B^{\prime}, C^{\prime}$ the midpoints of $B C, C A, A B$ respectively. Since the reflection in the origin may be described by the formulas $x^{\prime}=-x$, $y^{\prime}=-y$, it follows that the reflection in $(a, b)$ is given by the formulas $x^{\prime}=-x+2 a, y^{\prime}=-y+2 b$. Hence it follows from I.3, Theorem 1, that $A^{\prime}=(1 / 2,1 / 2), B^{\prime}=(0,1 / 2), C^{\prime}=(1 / 2,0)$, considering that the characteristic of $F$ is different from 2 . The equations of the three medians of the triangle are therefore

$$
y=x \cdot 1, \quad y=x(-1 / 2)+1 / 2, \quad y=x(-2)+1 .
$$

If the characteristic of the plane is 3 , then $-2=-1 / 2=1$; and the three medians are parallel, since $F$ and the plane have the same characteristic. If the characteristic is not 3 , then the common point of the three medians is $(1 / 3,1 / 3)$. This completes the proof. 
From the proof we see that the medians divide each other in the ratio $1: 2$, if the characteristic is not 3 . Here we say that the point $U$ divides $V W$ in the ratio $1: 2$, if $U$ is the midpoint of $V Z$ where $Z$ is the uniquely determined point such that $W$ is the midpoint of $U Z$.

\section{Chapter II. Orthogonaltty}

II:1. Altitudes and perpendicular bisectors of a tringle. Throughout the remainder of this investigation we shall assume without restating this assumption that we are dealing with an affine plane possessing a midpoint relation $X \cdot Y=Z$ which satisfies the postulates I to $\mathrm{V}$ of II.1. We are adding now an orthogonality relation, saying that certain lines are perpendicular to each other, in symbols $a \perp b$. This relation will be subject to the following postulates.

0.1. To every line a there exists a line $b$ such that $a \perp b$.

0.2. $a \perp b$ if, and only if, $b \perp a$.

0.3. If $a \perp b$, then a $\| a^{\prime}$ is a necessary and sufficient condition for $a^{\prime} \perp b$.

In short, an orthogonality relation constitutes an involutorial correspondence between the pencils of parallel lines.

THEOREM 1. The following properties imply each other.

0.4. The altitudes of a triangle are copunctual.

0.5. The perpendicular bisectors of a triangle are copunctual.

Proof $\left({ }^{\circ}\right)$. Suppose that the three points $A, B, C$ are not collinear. Denote by $A^{\prime}, B^{\prime}, C^{\prime}$ the midpoints of $B C, C A, A B$ respectively. Then it follows from I.5, Theorem 2, that the lines $A+B, B+C, C+A$ are parallel to the lines $A^{\prime}+B^{\prime}, B^{\prime}+C^{\prime}, C^{\prime}+A^{\prime}$ respectively. The perpendicular bisectors of the triangle $A, B, C$ are therefore the altitudes of the triangle $A^{\prime}, B^{\prime}, C^{\prime}$; and thus 0.5 is a consequence of 0.4 .

To prove that 0.4 is a consequence of 0.5 denote by $a, b, c$ the lines through the points $A, B, C$ respectively which are parallel to the lines $B+C, C+A$, $A+B$ respectively. Then it follows from I.5, Theorem 2 , that $A=(a b) \circ(a c)$, $B=(b c) \circ(b a), C=(c a) \circ(c b)$; and thus the altitudes of the triangle $A, B, C$ are the perpendicular bisectors of the triangle $b c, c a, a b$ so that 0.4 is a consequence of 0.5 .

If we say that parallel lines and only parallel lines are perpendicular, then the postulates 0.1 to 0.3 are satisfied. We shall always exclude this trivial possibility which is, for example, incompatible with 0.4 .

If the orthogonality relation is not trivial, then it is possible (because of I.4, Lemma 2) to introduce coordinates in such a way that the lines $x=$ const. are perpendicular to the lines $y=$ const. In the future we shall consider only such rectangular systems of coordinates.

Suppose now that the plane is described by a rectangular system of co-

( $\left.{ }^{9}\right)$ This proof is essentially the proof given in high school texts. 
ordinates and that the coordinates are numbers in the right distributive Cartesian number system $F$ of characteristic not 2. Then all the lines perpendicular to the line $y=x r, r \neq 0$, are parallel and they are all perpendicular to every line $y=x r+s$. Furthermore they are not of the form $x=$ const. or $y=$ const. Thus there exists to every number $r \neq 0$ in $F$ one and only one number $r^{*} \neq 0$ in $F$ such that:

(1) the line $y=x r+s$ is perpendicular to the line $y=x r^{*}+t ; r^{* *}=r$;

(2) perpendicularity of the line $y=x r+s$ and of the line $a$ implies that the line $a$ is of the form $y=x r^{*}+t$.

We note that the function $r^{*}$ depends on the system of coordinates and not only on the perpendicularity relation under consideration. A change of coordinates may effect a change in the system $F$; but even if it does not do so, it may effect a change in the function $r^{*}$. On the other hand, the function $r^{*}$ completely determines perpendicularity.

THEOREM 2. If the plane is characterized by a rectangular system of coordinates, taken from the right distributive Cartesian number system $F$, and if the perpendicularity relation is determined by the function $r^{*}$, then the following properties imply each other.

0.4 . The altitudes of a triangle are copunctual.

0.6. If no three of the four points $A, B, C, D$ are collinear, if $A+B \| C+D$, $A+C \| B+D, A+B \perp A+C$, and if $U$ is a point different from $A, B, C, D$ such that $B+U \perp C+U$, then $A+U \perp D+U$.

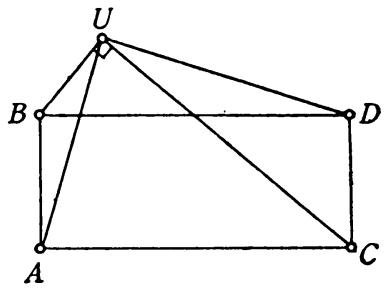

Condition 0.6

FIG. 5

0.7. $F$ is an ordinary commutative $\left({ }^{10}\right)$ and associative field and $r r^{*}=s s^{*}$ for $r \neq 0, s \neq 0$.

REMARK. The importance of 0.6 becomes apparent in the theory of circles and in the theory of congruence. See II.4 and III.2 below.

The proof will be effected in a number of steps.

(2.1) Both 0.4 and 0.6 imply the following property of the function $r^{*}$.

$\left(2.1^{\prime}\right)$ If $r, s, u, v$ are numbers different from 0 in $F$, then $r u=s v$ and $r v^{*}=s u^{*}$ imply each other.

$\left({ }^{10}\right)$ Schur $[1$, p. 207] has shown that 0.4 implies a certain special case of the Theorem of Pappus which suffices to prove commutativity of multiplication. 
Proof. Because of $r^{* *}=r$ it suffices to show that $r v^{*}=s u^{*}$ is a consequence of $r u=s v$, if 0.4 or 0.6 holds true. This is certainly the case if $r=s$, since then we would have $u=v$. Thus we assume $r \neq s$. Likewise we may assume $u \neq v$.

Put $t=-(r u)=-(s v)$ and assume the validity of 0.4 . The triangle with the vertices $(0, t),(r, 0)$ and $(s, 0)$ is formed by the lines $y=0, y=x u+t$, $y=x v+t$. Its altitudes are the lines $x=0, y=x u^{*}-s u^{*}, y=x v^{*}-r v^{*}$. These are three different lines which have one and only one point in common, so that $s u^{*}=r v^{*}$, as we intended to prove.

If $r u$ were equal to $s u^{*}$, then $u^{*}$ would be equal to $v, v^{*}$ equal to $u$ and hence $r v^{*}=s u^{*}$. Thus we may assume now that $r u \neq s u^{*}$ and that 0.6 holds true. Consider the points $A=\left(0, r u-s u^{*}\right), B=\left(r-s, r u-s u^{*}\right), C=(0,0)$, $D=(r-s, 0)$ which clearly form a rectangle; and the point $U=(r, r u)$ which is certainly different from these four points. The line $B+U$ is given by the equation $y=x u^{*}+r u-r u^{*}$, as follows from right distributivity and the commutative law of addition; and the line $C+U$ is given by $y=x u$. These two lines are clearly perpendicular. Hence we deduce from 0.6 the orthogonality of the lines $A+U$ and $D+U$. But $D+U$ is given by the equation $y=x v$ $+(s-r) v$, as follows from right distributivity, commutativity of addition and $s v=r u$. The line $y=x v^{*}-r v^{*}+r u$ is clearly perpendicular to $D+U$ and contains $U$. Hence $A$ must be on this line too, showing that $r u-s u^{*}=r u-r v^{*}$ or $r v^{*}=s u^{*}$, as we desired to prove.

(2.2) If $h$ and $k$ are numbers different from 0 in $F$, and if $\left(2.1^{\prime}\right)$ is satisfied by the function $r^{*}$, then

$$
h h^{*}=k k^{*} \quad \text { and } \quad h k=k h .
$$

REMARK. We denote by $c$ the common value of all the products $r r^{*}$. This number $c$ which we term the constant of orthogonality is different from 0 and depends on the rectangular system of coordinates selected.

Proof. Let $r=-v, u=-1, s=1$. Then $r u=v=1 v=s v$, and hence it follows from $\left(2.1^{\prime}\right)$ that $-v v^{*}=r v^{*}=s u^{*}=(-1)^{*}$ and consequently we have $v v^{*}=(-1)(-1)^{*}$, proving our first contention.

There exists one and only one number $z$ such that $h k=z h$. These numbers $h, k, z$ are different from 0 . Hence it follows from $\left(2.1^{\prime}\right)$ that $h h^{*}=z k^{*}$. But we have already shown that $k k^{*}=h h^{*}$. Hence $k=z$ because of the absence of zero-divisors in $F$, proving $h k=k h$.

For the enunciation of the next lemma it will be convenient to introduce some notations. If $F$ is any Cartesian number system, and if $e$ is a number different from 0 in $F$, then there exists a Cartesian number system $F_{\theta}$ with unit element $1_{e}$ and 0 -element 0 such that $(0,0)=(0,0),(0, e)=\left(0,1_{\epsilon}\right)$, $(e, 0)=\left(1_{e}, 0\right)$, as follows from I.4, Lemma 2. Then the plane over $F$ and the plane over $F_{e}$ are the same, both systems of coordinates have the same $x$-axis and the same $y$-axis. Since the additive group of the Cartesian number system is just the group of translations in the direction of the $y$-axis, it follows that 
the addition groups of $F$ and $F_{\theta}$ are identical, and that these systems differ only by the definition of multiplication. We denote the product of two elements $m$ and $n$ in $F$ by $m n$ and in $F_{e}$ by $m o_{e} n$. The line $y=x r$ passes through the points $(0,0)$ and $(e, e r)=\left(1_{e}, e r\right)$ so that this line coincides with the line $y=x \circ_{e}(e r)$. Thus we obitain the formula

$$
s r=s o_{e}(e r)
$$

connecting the multipiication in $F$ and the multiplication in $F_{e}$.

(2.4) If multiplication is commutative in every $F_{e}$, then it is associative in $F$ (and in every $F_{s}$ ).

Proof. From (2.3) and the hypotheses we deduce

$$
r(e s)=(e s) r=(e s) \circ_{e}(e r)=(e r) \circ_{e}(e s)=(e r) s=(r e) s,
$$

as was to be shown.

Proof that 0.7 is a consequence of 0.4 and of 0.6 . If 0.4 or 0.6 is satisfied by the orthogonality relation, then $\left(2.1^{\prime}\right)$ holds in every $F_{s}$ for $e \neq 0$. Thus it follows from (2.2) that every $F_{e}$ is commutative; and we infer from (2.4) that $F$ is associative. But $F$ is right distributive and hence $F$ is an ordinary commutative field. That the function $r^{*}$ (in $F$ ) meets the requirement $r r^{*}=s s^{*}$ is a consequence of (2.2). Thus we have shown that 0.7 is a consequence of 0.4 as well as of 0.6 .

If $F$ is an ordinary commutative field of characteristic not 2, if $c$ is a number, not 0 , in $F$, and if we define perpendicularity in the plane over $F$ by the rules:

(1) the lines $x=$ const. and the lines $y=$ const. are perpendicular;

(2) if $r \neq 0$, then the lines $y=x r+s$ and the lines $y=x c r^{-1}+t$ are perpendicular; then it is clear that this definition of orthogonality meets the requirements 0.1 to 0.3 .

(2.5) If 0.7 is satisfied, if $A=(u, v), B=(-u,-v)$ are points different from $(0,0)$, and if $P=(x, y)$ is different from $A$ and $B$, then the following condition is necessary and sufficient for $A+P \perp B+P$ :

$$
y^{2}-v^{2}=c\left(x^{2}-u^{2}\right)
$$

where $c \neq 0$ is the constant of orthogonality.

Proof. If $u=x$, then $y=-v$ is necessary and sufficient for both $\left(2.5^{\prime}\right)$ and $A+P \perp B+P$, since $P \neq A$ and $P \neq B$. If $v=y$, then $x=-u$ is necessary and sufficient for both $\left(2.5^{\prime}\right)$ and the orthogonality of $A+P$ and $B+P$.

If a line is given by the equation $y=x r+s$, then $r$ is its slope. If $u \neq \pm x$ and $v \neq \pm y$, then the slope of $A+P$ is $(y-v) /(x-u)$ and the slope of $B+P$ is $(y+v) /(x+u)$. Thus the perpendicularity of $A+P$ and $B+P$ is equivalent to

$$
\frac{y-v}{x-u} \frac{y+v}{x+u}=c
$$


and this equation is equivalent to $\left(2.5^{\prime}\right)$.

Proof that 0.6 is a consequence of 0.7. Assume that no three of the four points $A, B, C, D$ are collinear, that $A+B\|C+D, A+C\| B+D, A+B \perp A+C$, that the point $U$ is different from $A, B, C, D$ and that $A+U \perp D+U$. Since the group of translations is transitive, we may assume without loss of generality that the diagonals of the parallelogram (rectangle) $A, B, C, D$ meet( ${ }^{11}$ ) in the origin $(0,0)$. Then $A=(u, v), B=(r, s), C=(-r,-s), D=(-u,-v)$, since $(0,0)$ is the midpoint of both $A D$ and $B C$. If $U=(x, y)$, then it follows from our hypotheses and (2.5) that

$$
\begin{aligned}
& y^{2}-v^{2}=c\left(x^{2}-u^{2}\right) \\
& s^{2}-v^{2}=c\left(r^{2}-u^{2}\right) ;
\end{aligned}
$$

and by subtraction we obtain $y^{2}-s^{2}=c\left(x^{2}-r^{2}\right)$. But it follows from (2.5) that this last equation is equivalent to perpendicularity of $U+B$ and $U+C$, proving 0.6.

Proof that 0.4 is a consequence of 0.7 . From the equivalence of 0.6 and 0.7 it follows that every rectangular system of coordinates meets the requirement 0.7 if at least one of them satisfies 0.7 .

Suppose now that the three points $A, B, C$ are not collinear. If $A+B$ $\perp B+C$, then the three altitudes of the triangle $A, B, C$ meet in $B$. Thus we may assume that $A, B, C$ is not a rectangular triangle.

It is impossible that the three lines $A+B, B+C, C+A$ are all self-perpendicular. For no two of them are parallel, and there exist at most two pencils of parallel lines which are self-perpendicular. The latter fact may be verified as follows. If $c$ is the constant of orthogonality in the rectangular system of $x=y$-coordinates, then self-perpendicularity of the line $y=x r+s$ is equivalent to $r^{2}=c$. But this equation has either two or no solutions. Thus we may assume that the line $A+B$ is not self-perpendicular.

Now it follows from an earlier remark that we may assume without loss of generality,

$$
A=(0,0), B=(0, r), C=(s, t) \text { where } r \neq t, s t \neq 0,
$$

since the three points $A, B, C$ are not collinear and do not form a rectangular triangle. Then the lines $A+B, B+C, C+A$ are given by the equations $x=0$, $y=x(t-r) s^{-1}+r, y=x t s^{-1}$ respectively; and the three altitudes through $A, B, C$ are given by $y=x s(t-r)^{-1} c, y=x s t^{-1} c+r, y=t$ respectively; and these three lines meet in the point $\left(t(t-r) s^{-1} c^{-1}, t\right)$. Here $c$ signifies throughout the constant of orthogonality connected with the selected system of rectangular coordinates. This completes the proof.

\section{COROLLARY 1. Suppose that the orthogonality relation satisfies the postulates}

(i1) That the diagonals of this parallelogram meet in a point (are not parallel) is a consequence of Fano's axiom; see I.2, Remark to Theorem 3. 
0.1 to 0.4 . Then either no line is self-perpendicular or there exist exactly two different pencils of parallel lines which are self-perpendicular.

Proof. There exists one and essentially only one field $F$ of characteristic different from 2 such that the plane is the plane over $F$. There exists a rectangular system of coordinates and a constant of orthogonality $c \neq 0$ in $F$ such that the lines $y=x r$ and $y=x s$ are perpendicular if, and only if, $r s=c$. Thus there exist self-perpendicular lines if, and only if, $c$ is a square in $F$. These facts are consequences of Theorem 2 , and from these facts one readily deduces Corollary 1 as well as the following statement.

Corollary 2. Suppose that $F$ is a commutative field of characteristic different from 2 and that $c \neq 0$ belongs to $F$. The orthogonality relation defined by the constant of orthogonality in the affine plane over $F$ admits self-perpendicular lines if, and only if, $c$ is a square in $F$.

\section{ApPendiX: Collinearity of the SIGNificant POINTS OF A tRIANGLE}

The validity of the so-called affine specialization of Desargues' Theorem is a well known consequence of Condition 0.7. We are going to assume throughout this appendix that the affine plane under consideration is of characteristic different from 2 and satisfies the affine specialization of Desargues' Theorem. Using the results of the first chapter it follows that there exists one and only one midpoint relation satisfying postulates I to V. We assume furthermore that an orthogonality relation has been defined which meets the requirements 0.1 to 0.3 .

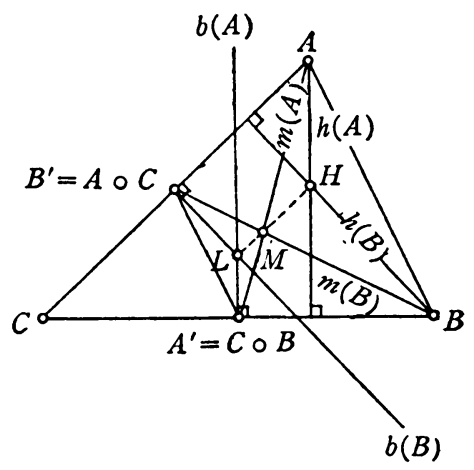

Fig. 6

If the three points $A, B, C$ are not collinear, then we denote by $A^{\prime}, B^{\prime}, C^{\prime}$ the midpoints of $B C, C A$ and $A B$ respectively, by $h(X)$ the altitude through $X$, by $b(X)$ the perpendicular bisector through $X^{\prime}$ and by $m(X)$ the median through $X$ and $X^{\prime}$. It is a consequence of I.6, Theorem, that the three medians meet in a point $M$ (note that $M$ is an ideal point if, and only if, the character- 
istic of the plane is 3$)$. It is readily seen that $h(A)$ and $h(B)$ meet in a well determined finite point and that $b(A)$ and $b(B)$ do likewise.

$M, h(A) h(B)$ and $b(A) b(B)$ are collinear.

The proof of this fact is an adaptation of an argument given by Schur [1, p. 91]. Consider the triangles $A, B, h(A) h(B)$ and $A^{\prime}, B^{\prime}, b(A) b(B)$. Since $h(X)$ and $b(X)$ are perpendicular to the same side of the triangle $A, B, C$, they are parallel; and the parallelism of $A+B$ and $A^{\prime}+B^{\prime}$ is a consequence of I.5, Theorem 1. Thus corresponding sides of these two triangles have been shown to be parallel. Applying Desargues' Theorem-which may be applied whenever our assertion is not trivially satisfied-we infer the perspectivity of the two triangles $A, B, h(A) h(B)$ and $A^{\prime}, B^{\prime}, b(A) b(B)$, proving our contention.

From the fact just proved one now readily deduces the following improvement upon II.1, Theorem 1.

Suppose that the three points $A, B, C$ are not collinear. Then the three altitudes of this triangle are copunctual if, and only if, the perpendicular bisectors are copunctual. If the altitudes of the triangle meet in a point $H$ and the perpendicular bisectors meet in a point $L$, then $H, L$ and $M$ are collinear $\left({ }^{12}\right)$.

II.2. Types of orthogonality relations. It is apparent from the results of II. 1 that an affine plane admits of an orthogonality relation satisfying 0.1 to 0.4 if, and only if, it is a plane over an ordinary commutative field of characteristic different from 2. But we shall show later on that it is always possible to define orthogonality in a number of different ways. Not all of these definitions of orthogonality will be essentially different. To give a precise meaning to this term we define: two orthogonality relations in the same affine plane are equivalent, if there exists an affinity of the plane which maps pairs of lines perpendicular under the first orthogonality relation upon pairs of lines perpendicular under the second orthogonality relation. Here an affinity is a $1: 1$ correspondence between the points of the affine plane which maps collinear points upon collinear points. Clearly affinities preserve parallelism; and hence it follows from I.2, Theorem 2, that the midpoint relation is invariant under affinities.

THEOREM 1. If $R$ and $R^{\prime}$ are orthogonality relations in the same affine plane, and if postulates 0.1 to 0.4 are satisfied by $R$ and $R^{\prime}$, then the following properties of $R$ and $R^{\prime}$ imply each other.

(1) $R=R^{\prime}$.

(2) There exist two different pairs of pencils of parallel lines which are perpendicular under both $R$ and $R^{\prime}$.

(3) There exist a system of coordinates which is rectangular under $R$ and

${ }^{(12)}$ Baldus [1] has shown that this theorem is in a certain sense characteristic for Euclidean geometry. 
under $R^{\prime}$ and which leads to the same constant of orthogonality under $R$ and under $R^{\prime}$.

Proof. It is obvious that (1) implies (2). If (2) holds true, then there exist two different pairs of lines $a, a^{\prime}$ and $b, b^{\prime}$ which are both perpendicular under both definitions, and which meet in one point. We distinguish two cases.

Case 1. $a=a^{\prime}$ and $b=b^{\prime}$. Then $a \neq b$. We choose a system of $R$-rectangular coordinates such that $(0,0)=a b$. Then it follows from II.1, Corollary 2 , that the equations of these lines $a, b$ are $y=x r$ and $y=x(-r)$ respectively and that $c=r^{2}$ is the constant of orthogonality belonging to this system of rectangular coordinates and to the relation $R$.

We deduce from I.4, Lemma 2 , and from II.1, Corollary 1 , the existence of a system of $R^{\prime}$-rectangular coordinates whose $y$-axis is the same as the $y$-axis of the first system of coordinates and which has the same origin. Since these two systems of coordinates describe the same affine plane, the coordinates $x, y$ of the first system and the coordinates $x^{\prime}, y^{\prime}$ of the second system are connected by the following formulas $\left({ }^{13}\right)$ :

$$
x=x^{\prime f} s, \quad y=x^{\prime f} t+y^{\prime f} u,
$$

where $s u \neq 0$ and where $f$ is an automorphism of the field $F$ from which the coordinates are taken.

The equation of the line $y=x( \pm r)$ in the $x^{\prime}=y^{\prime}$-system is then

$$
y^{\prime}=x^{\prime}(s( \pm r)-t)^{r^{-1} u^{-f^{-1}}} \text {. }
$$

But these two lines are self-perpendicular under $R^{\prime}$; and hence it follows from 0.7 that

$$
(s r-t)^{2} u^{-2}=(s(-r)-t)^{2} u^{-2} \text { or } \quad-s r t=s r t .
$$

But $s$ and $r$ are both different from 0 , and the characteristic of $F$ is not 2 . Hence $t=0$. Thus we have shown:

There exists a pair of lines which are not parallel, though they are perpendicular both under $R$ and under $R^{\prime}$; and this reduces case 1 to case 2 .

Case 2. At least one of the pairs of lines is not self-perpendicular. In this case there exists a system of coordinates which is rectangular under both $R$ and $R^{\prime}$. There exists furthermore a pair of lines $y=x r$ and $y=x s$ which is not parallel to the $x$ - or the $y$-axis and which is perpendicular under both $R$ and $R^{\prime}$. But then it follows from 0.7 that the constant of orthogonality belonging to this system of coordinates is both under $R$ and under $R^{\prime}$ just the number $r s$, proving that (2) implies (3). That (3) implies (1) is obvious; and this completes the proof.

THEOREM 2. If $R$ and $R^{\prime}$ are orthogonality relations in the same affine plane, if both $R$ and $R^{\prime}$ satisfy 0.1 to 0.4 , and if their constants of orthogonality in

(13) For a proof see, for example, Kamke [1, p. 145-147] or Reidemeister [1]. 
suitable rectangular systems of coordinates are $c$ and $c^{\prime}$ respectively, then the following condition is necessary and sufficient for equivalence of $R$ and $R^{\prime}$ :

There exists an automorphism $f$ of the field $F$ of coordinates and a number $s \neq 0$ in $F$ such that

$$
c=c^{\prime f} s^{2} .
$$

Proof. Suppose that a system of $R$-rectangular coordinates be given and that the constant of orthogonality belonging to this system of $x=y$-coordinates is $c$. If $a$ is an affinity of the plane which maps the point $(x, y)$ upon the point $\left(x^{\prime}, y^{\prime}\right)$, then an orthogonality relation $R^{a}$ is defined by the rule:

The lines $u$ and $v$ are perpendicular under $R$ if, and only if, the lines $u^{a}$ and $v^{a}$ are perpendicular under $R^{a}$.

Clearly $R^{a}$ meets the requirements 0.1 to 0.4 , since these postulates are satisfied by $R$.

We start by discussing some special types of affinities.

Type 1. $x^{\prime}=x+h, y^{\prime}=y+k$. Then the $x=y$-coordinates are rectangular for both $R$ and $R^{a}$ and $c$ is the constant of orthogonality of $R^{a}$ in this system of coordinates.

Type 2. $x^{\prime}=x^{f}, y^{\prime}=y^{f}$ for $f$ an automorphism of $F$. Then the $x=y$-coordinates are rectangular for both $R$ and $R^{a}$ and $c^{f}$ is the constant of orthogonality of $R^{a}$ in this system of coordinates.

Type 3. $x^{\prime}=x r, y^{\prime}=y s$ for $r s \neq 0$. Then the $x=y$-coordinates are rectangular for both $R$ and $R^{a}$. The line $y=x$ is mapped by $a$ upon the line $y^{\prime}=x^{\prime} s r^{-1}$. The line $y=x c$ which is perpendicular to $y=x$ under $R$ is mapped by $a$ upon the line $y^{\prime}=x^{\prime} s r^{-1} c$. Hence it follows that the constant of orthogonality of $R^{a}$ in the system of $x=y$-coordinates is just $c\left(s r^{-1}\right)^{2}$.

Type 4. $x^{\prime}=x a+y, y^{\prime}=x b+y$ for $a b=\dot{c}, a \neq b$. Since the lines $y=x(-a)$ and $y=x(-b)$ are perpendicular under $R$, it follows that the $x^{\prime}=y^{\prime}$-coordinates are rectangular for both $R$ and $R^{a}$. The line $x=0$ is mapped by $a$ upon the line $x^{\prime}=y^{\prime}$ and the line $y=0$ is mapped by $a$ upon $y^{\prime} / b=x^{\prime} / a$. Thus the constant of 'orthogonality derived from the $x^{\prime}=y^{\prime}$-coordinates is $b / a=c / a^{2}$.

Every change from one system of $R$-rectangular coordinates to another system of $R$-rectangular coordinates may be effected by a succession of transformations of the types 1 to 4 just discussed.

The orthogonality relations $R$ and $R^{\prime}$ are equivalent if, and only if, there exists a system of $R$-rectangular coordinates and a system of $R^{\prime}$-rectangular coordinates such that the corresponding constants of orthogonality are the same. Combining the remarks in the last two paragraphs with the discussion of the types 1 to 4 , one now readily verifies the theorem.

REMARK. If $F$ is an ordinary commutative field of characteristic different from 2, and if $c \neq 0$ is a number in $F$, then there exists one and essentially only one extension $F\left(c^{1 / 2}\right)$; and the quadratic extensions of $F$ which are determined by $c$ and $c^{\prime}$ are essentially the same if, and only if, $c=c^{\prime} s^{2}$ for $s$ 
in $F$. Two extensions $U$ and $V$ of $F$ are similar if there exists an isomorphism of $U$ upon $V$ which maps $F$ upon itself. Now it is possible to restate the condition of Theorem 2 as follows.

The quadratic extensions of $F$ determined by the numbers $c$ and $c^{\prime}$ respectively are similar.

Thus we have obtained a 1:1 correspondence between the classes of similar quadratic extensions of $F$ and the classes of equivalent orthogonality relations satisfying 0.1 to 0.4 .

If no three of the four points $A, B, C, D$ are collinear, if $A+B \| C+D$, $A+C \| B+D, A+B \perp A+C$, then the points $A, B, C, D$ form a rectangle; and if the diagonals in this rectangle are perpendicular, then it is called a square.

If $R$ is an orthogonality relation satisfying 0.1 to 0.4 , and if $c$ is the constant of orthogonality determined by $R$ and some system of rectangular coordinates, then it is readily seen that

$$
c=-s^{2} \text { for } s \text { in } F
$$

is a necessary and sufficient condition for the existence of a square. Applying Theorem 2 we obtain the following fact.

COROLLARY 1. In an affine plane over an ordinary commutative field of characteristic different from 2 there exists one and essentially only one orthogonality relation which satisfies 0.1 to 0.4 such that there exists a square.

Finally we state the following fact which is an immediate consequence of Theorem 2 and II.1, Corollary 2.

COROLLARY 2. There exists one and essentially only one orthogonality relation without self-perpendicular lines which satisfies the postulates 0.1 to 0.4 if, and only if, the following three conditions are satisfied.

(a) The plane is the affine plane over an ordinary commutative field $F$ (of characteristic different from 2).

(b) -1 is not a square in $F$.

(c) If $s$ is not a square in $F$, then $-s$ is a square.

II.3. The bisectors of angles. All the orthogonality relations considered in this section will satisfy 0.1 to 0.3 and will have the property that there do not exist self-perpendicular lines.

Suppose now that $u, v, w$ are three different lines through the point $P$, and that they meet the following requirement.

(B) If $W \neq P$ is a point on $w$, if $w^{\prime}$ is a line perpendicular to w through $W$, then $w^{\prime}$ is not parallel to either $u$ or $v$ and $W$ is the midpoint of $\left(w^{\prime} u\right)\left(w^{\prime} v\right)$.

Then we term the line $w$ a bisector of the angle $(u, v)$.

We note that a bisector of the angle $(u, v)$ is at the same time a bisector of the angle $(v, u)$. It should be understood that an angle is nothing but a pair of lines. 
THEOREM 1. The validity of postulate 0.4 is equivalent to the validity of the following three conditions.

(i) If the three points $A, B, C$ are not collinear and do not form a rectangular triangle, if $A^{\prime}, B^{\prime}, C^{\prime}$ are the feet of the altitudes of the triangle $A, B, C$ through $A, B, C$ respectively, then $A^{\prime}, B^{\prime}, C^{\prime}$ are not collinear and the line $A+A^{\prime}$ is a bisector of the angle $\left(A^{\prime}+C^{\prime}, A^{\prime}+B^{\prime}\right)$.

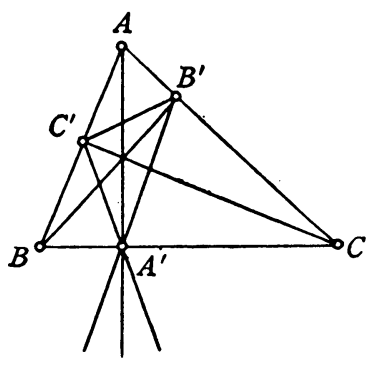

Condition (i)

FIG. 7

(ii) If the three points $A, B, C$ are not collinear, if $u$ is a bisector of the angle $(A+B, A+C)$ and $v$ a bisector of the angle $(B+A, B+C)$, then $C+u v$ is a bisector of the angle $(C+A, C+B)$.

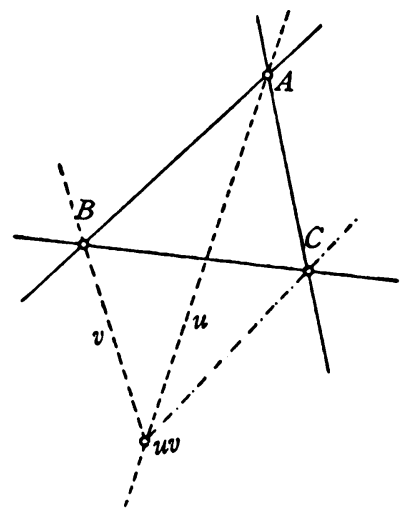

Condition (ii)

FIG. 8

(iii) If $w$ and $w^{\prime}$ are different bisectors of the angle $(u, v)$, then $w \perp w^{\prime}$.

Proof. We assume first the validity of the conditions (i) to (iii). If the three points $A, B, C$ form a rectangular triangle, then its altitudes have certainly a point in common; and thus we may assume without loss of generality that the points $A, B, C$ are not collinear, but do not form a rectangular triangle. Denote by $A^{\prime}, B^{\prime}, C^{\prime}$ the uniquely determined points on $B+C, C+A$, 
$A+B$ respectively such that $A+A^{\prime} \perp B+C, B+B^{\prime} \perp C+A, C+C^{\prime} \perp A+B$. Then we deduce from (i) that $A+A^{\prime}$ is a bisector of the angle $\left(A^{\prime}+C^{\prime}\right.$, $\left.A^{\prime}+B^{\prime}\right)$, that $B+B^{\prime}$ is a bisector of the angle $\left(B^{\prime}+C^{\prime}, B^{\prime}+A^{\prime}\right)$ and that $C+C^{\prime}$ is a bisector of the angle $\left(C^{\prime}+A^{\prime}, C^{\prime}+B^{\prime}\right)$. It follows from (ii) that $w=C^{\prime}+\left(A+A^{\prime}\right)\left(B+B^{\prime}\right)$ is a bisector of the angle $\left(C^{\prime}+A^{\prime}, C^{\prime}+B^{\prime}\right)$. If $w$ were different from $C+C^{\prime}$, then we would infer from (iii) that $w \perp C+C^{\prime}$. Hence we would have $w=A+B$. Thus $\left(A+A^{\prime}\right)\left(B+B^{\prime}\right)$ would be on $A+B$. But $A+B$ meets $A+A^{\prime}$ in $A$ and $B+B^{\prime}$ in $B$; and thus it would follow that $A=\left(A+A^{\prime}\right)\left(B+B^{\prime}\right)=B$, an impossibility proving that $w=C+C^{\prime}$. Hence $C+C^{\prime}$ passes through $\left(A+A^{\prime}\right)\left(B+B^{\prime}\right)$, proving that postulate 0.4 is a consequence of conditions (i), (ii), (iii).

Suppose next that 0.4 be valid. Then 0.7 is satisfied too, as follows from II.1, Theorem 2. Thus the affine plane is the plane over an ordinary commutative field of characteristic different from 2. If we consider a system of rectangular $x=y$-coordinates, then orthogonality is determined by the constant of orthogonality $c$. We prove some lemmas.

(1.1) The line $y=x s$ is a bisector of the angle $(y=0, x=0)$ if, and only if, $s^{2}=-c$.

Consider the point $(h, h s), h \neq 0$, on the line $y=x s$. The line perpendicular to $y=x s$ passing through $(h, h s)$ is given by $y=x c s^{-1}-h c s^{-1}+h s$; and this line meets the $y$-axis in $\left(0, h s-h c s^{-1}\right)$, the $x$-axis in $\left(-h s^{2} c^{-1}+h, 0\right)$. The point $(h, h s)$ is the midpoint between these points if, and only if,

$$
2 h=-h s^{2} c^{-1}+h \text { and } 2 h s=h s-h c s^{-1} ;
$$

and these two equations are both equivalent to $s^{2}=-c$.

(1.2) The line $y=x s$ is a bisector of the angle $(y=0, y=x r)$ if, and only if, $s^{2} r-2 s c+r c=0(r \neq 0)$.

Consider again the point $(h, h s)$ on the line $y=x s$ and the line $y=x c s^{-1}$ $-h c s^{-1}+h s$ which passes through $(h, h s)$ and is perpendicular to $y=x s$. This line meets the $x$-axis in $\left(-h s^{2} c^{-1}+h, 0\right)$ and the line $y=x r$ in $\left(h\left(s^{2}-c\right)(r s-c)^{-1}\right.$, $\left.h r\left(s^{2}-c\right)(r s-c)^{-1}\right)$. The point $(h, h s)$ is the midpoint between these two points if, and only if,

$$
2 h=-h s^{2} c^{-1}+h+h\left(s^{2}-c\right)(r s-c)^{-1} \quad \text { and } \quad 2 h s=h r\left(s^{2}-c\right)(r s-c)^{-1} \text {. }
$$

The latter of these equations is clearly equivalent to the equation $s^{2} r-2 s c+r c$ $=0$; and the first of these two equations is readily seen to be satisfied whenever the second is.

(1.3) The $y$-axis is a bisector of the lines $y=x r$ and $y=x s$ for $r \neq s$ and $r s \neq 0$ if, and only if, $r=-s$.

This is immediately verified. 
If $w$ and $w^{\prime}$ are different bisectors of the angle $(u, v)$, then we may assume without loss of generality that $u$ is the $x$-axis, that $v$ is either the $y$-axis or the line $y=x r$, and that $w$ and $w^{\prime}$ are the lines $y=x s$ and $y=x s^{\prime}$ respectively. Then we deduce from (1.1) and (1.2) that $s s^{\prime}=c$, proving the validity of (iii).

If the three points $A, B, C$ are not collinear, and do not form a rectangular triangle, then we may, because of I.4, Lemma 2, choose the system of rectangular $x=y$-coordinates in such a way that $A=(s, 0), B=(r, 0)$, $C=(0, t)$, where $r s t \neq 0$ and $r \neq s$, and such that the foot of the altitude through $C$ is $C^{\prime}=(0,0)$. Then $A+C$ is given by $y=-x t s^{-1}+t$ and $B+C$ by $y=-x t r^{-1}+t$. The altitudes through $A$ and $B$ are therefore $y=-x r c t^{-1}+\operatorname{srct}^{-1}$ and $x=-x s c t^{-1}+r s c t^{-1}$, where $c$ is the constant of orthogonality belonging to the system of coordinates under consideration. The foot $A^{\prime}$ of the altitude through $A$ is therefore $\left(r\left(s r c-t^{2}\right)\left(r^{2} c-t^{2}\right)^{-1}, \operatorname{trc}(s-r)\left(t^{2}-r^{2} c\right)^{-1}\right)$ and the foot $B^{\prime}$ of the altitude through $B$ is likewise $\left(s\left(s r c-t^{2}\right)\left(s^{2} c-t^{2}\right)^{-1}, t s c(r-s)\left(t^{2}-s^{2} c\right)^{-1}\right)$. Hence the line $A^{\prime}+C^{\prime}$ is given by the equation $y=x t c(s-r)\left(t^{2}-s r c\right)^{-1}$ and the line $B^{\prime}+C^{\prime}$ is given by the equation $y=x t c(r-s)\left(t^{2}-s r c\right)^{-1}$. But $C+C^{\prime}$ is the $y$-axis; and hence it follows from (1.3) that $C+C^{\prime}$ is a bisector of the angle $\left(A^{\prime}+C^{\prime}, B^{\prime}+C^{\prime}\right)$, proving (i).

We precede the proof of condition (ii) by the proof of a lemma which does not make use of 0.4 .

(1.4) If the three points $A, B, C$ are not collinear, if $u$ is a bisector of the angle $(A+B, A+C)$ and $v$ a bisector of $(B+A, B+C)$, then $u$ and $v$ are neither parallel nor perpendicular.

Assume first that $u \| v$. Then there exists one and only one line $w$ through $C$ which is perpendicular to both $u$ and $v$. It follows from the definition of bisectors that $w$ is not parallel to $A+B$, that $w$ therefore meets $A+B$ in a point $W$ and that $u w$ is the midpoint of $W C$; similarly, $v w$ is the midpoint of $W C$. The midpoint being unique we deduce $u w=v w$, contradicting the supposed parallelism of $u$ and $v$, since $u$ and $v$ are certainly different.

Assume next that $u \perp v$. Then $u(B+C)$ is a well determined point $B^{\prime}$ and $u v$ is the midpoint of $A B^{\prime}$; and $v(A+C)$ is a well determined point $A^{\prime}$ and $u v$ is the midpoint of $B A^{\prime}$. Thus the diagonals of the quadrangle $A, A^{\prime}, B^{\prime}, B$ bisect each other. Since there exists only one point $X$ on the line $B+u v$ such that $u v$ is the midpoint of $B X$, it follows from I.2, Theorem 1 , that this quadrangle is a parallelogram, an impossibility since $A+A^{\prime}$ and $B+B^{\prime}$ are two different lines meeting in $C$. This completes the proof of the lemma.

We return now to the proof of the fact that (ii) is a consequence of 0.4 . If the three points $A, B, C$ are not collinear, if $u$ is a bisector of the angle $(A+B, A+C)$ and $v$ a bisector of the angle $(B+A, B+C)$, then we denote by $a$ the uniquely determined line through $A$ which is perpendicular to $u$ and by $b$ the uniquely determined line through $B$ which is perpendicular to $v$. We 
infer from (1.4) that the lines $u$ and $v$ are neither parallel nor perpendicular. The lines $u$ and $b$ are not parallel, since $u$ and $v$ are not perpendicular; and for the same reason $v$ and $a$ are not parallel. The lines $a$ and $b$ are not parallel, since $u$ and $v$ are not parallel. Thus $a b, a v, b u$ is a well determined triangle.

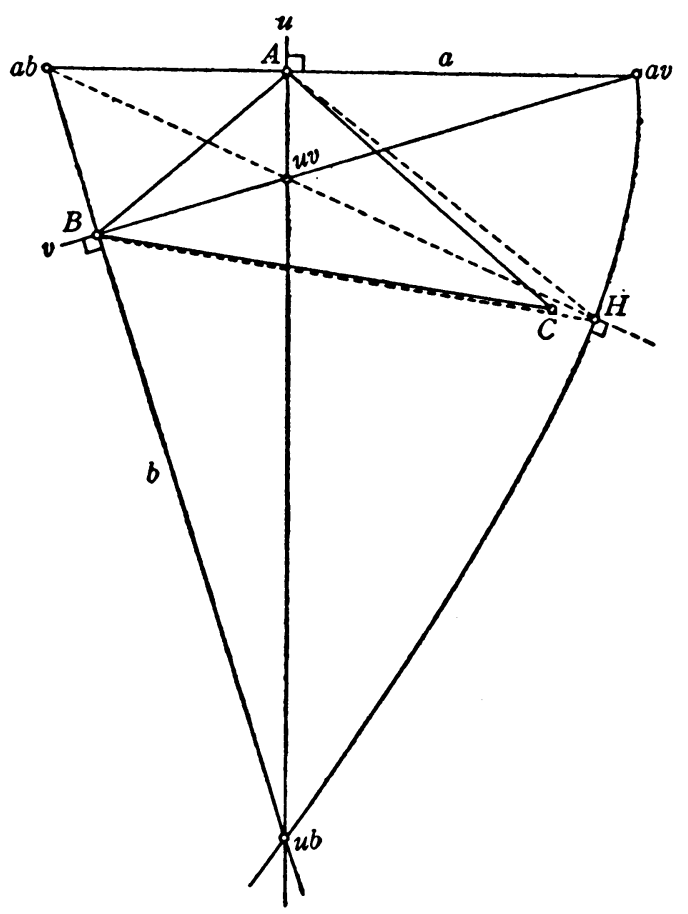

FIG. 9

Two of its altitudes are $u$ and $v$, the third one is a line $H+a b$ with footpoint $H$ on $b u+a v$. It is a consequence of 0.4 that the three altitudes pass through the same point, namely $u v$. But then it follows from property (i) which we already verified that $u$ is a bisector of the angle $(A+B, A+H), v$ a bisector of the angle $(B+A, B+H)$ and $a b+H$ a bisector of the angle $(H+B, H+A)$. But $u$ is a bisector of $(A+B, A+C)$ so that $A+H=A+C$, since there exists on the line $X+Y$ only one point $Z$ such that $Y$ is the midpoint of $X Z$; similarly, $B+H=B+C$. Hence $H=C$, showing that the line $a b+H=C+u v$ is a bisector of the angle $(C+A, C+B)$, as was to be shown.

From the proof, in particular (1.1) and (1.2), it is easy to deduce the following fact.

COROLLARY. If 0.4 is satisfied, then the following condition is necessary and sufficient for the existence of bisectors of every angle: 
(iv) If $c$ is the constant of orthogonality in some system of coordinates, then $-c$ is a square and the sum of any two squares is a square.

Since we assumed the absence of self-perpendicular lines, one may deduce from (iv) and II.1, Corollary 2, the reality of the field $F$.

The corollary makes it evident that the postulate 0.4 is weaker than the postulates-used in the treatments of absolute geometry $\left({ }^{14}\right)$ - ensuring the existence of reflections in lines which interchange a given pair of lines. Since $x^{\prime}=-x, y^{\prime}=y$ is a reflection in the $y$-axis, it is apparent that 0.4 ensures the existence of a reflection in every given line. But if the reflection in the line $w$ interchanges the different lines $u$ and $v$, then $w$ is a bisector of the angle $(u, v)$. Consequently the existence of a reflection in a line which interchanges the lines $u$ and $v$ implies the existence of a bisector of the angle $(u, v)$. But the corollary shows that the existence of bisectors of every angle is not a consequence of 0.4 .

II.4. Postulate 0.6 and the theory of circles. Suppose that in an affine plane there has been defined an orthogonality relation which meets the requirements 0.1 to 0.3 . If $D^{\prime}$ and $D^{\prime \prime}$ are different points, then we define the circle with diameter $D^{\prime} D^{\prime \prime}$ as the locus of the point of intersection of perpendicular lines through $D^{\prime}$ and $D^{\prime \prime}$. It therefore consists of $D^{\prime}, D^{\prime \prime}$ and all points $P$ for which $D^{\prime}+P \perp D^{\prime \prime}+P$.

The midpoint $Z$ of $D^{\prime} D^{\prime \prime}$ may be termed the center of this circle, and points $Q^{\prime} \neq Q^{\prime \prime}$ on the circle may be termed opposite points if $Z, Q^{\prime}, Q^{\prime \prime}$ are collinear. This is clearly the case if the points $D^{\prime}, Q^{\prime}, D^{\prime \prime}, Q^{\prime \prime}$ form a rectangle, and again if $Q^{\prime}+Q^{\prime \prime}=D^{\prime}+D^{\prime \prime}$. Suppose, further, that none of the lines $Q^{\prime}+Q^{\prime \prime}$, for $Q^{\prime}, Q^{\prime \prime}$ opposite points, are self-perpendicular. Then there arises the question whether every pair of opposite points forms a diameter, that is, whether the circle with diameter $Q^{\prime} Q^{\prime \prime}$ is always equal to the circle with diameter $D^{\prime} D^{\prime \prime}$. It is evident that Postulate 0.6 is necessary and sufficient to ensure this equality.

Assume now that 0.6 is satisfied by the orthogonality relation under consideration. Then we may assume without loss of generality that the center $Z$ of the circle is the origin $(0,0)$ of our system of rectangular coordinates. This implies $D^{\prime}=(r, s) \neq(0,0)$ and $D^{\prime \prime}=(-r,-s)$. If the constant of orthogonality belonging to our system of coordinates is $c$, then we deduce from II.1 (2.5) and II.1, Theorem 2, that:

The point $(x, y)$ belongs to the circle with diameter $D^{\prime} D^{\prime \prime}$ if, and only if,

$$
y^{2}-c x^{2}=s^{2}-c r^{2} .
$$

On the basis of this fact it is possible to develop the theory of circles and of rotations in the customary fashion. See in this respect in particular Bachmann $[1,2]$ and Bottema $[1,2,3]$. It should be noted, however, that the

(14) See footnote 2. 
postulates 0.1 to 0.4 do not ensure the existence of rotations mapping a certain pencil of parallel lines upon another preassigned pencil of parallel lines, as may be seen from the following simple example:

The plane is the affine plane over the field of rational numbers and the constant of orthogonality for a suitable system of rectangular coordinates is -1 . The circle given by the equation $x^{2}+y^{2}=1$ has the origin as its center and contains the opposite points $( \pm 1,0)$. But it does not contain any point of the line $y=x 2$, since 5 is not a square; and thus there does not exist a rotation around the origin mapping the $x$-axis upon the line $y=x 2$; and neither can there exist a reflection in a suitable line which interchanges these two lines.

\section{Chapter III. Congruence and orthogonality}

III.1. Vector equality. To avoid confusion we shall indicate the midpoint relation throughout this chapter by $X=Y \circ Z$, a notation that is in complete accordance with notations previously used, as follows from the results of I.2.

The discussion of vector equality which we are going to give now will not provide new results. Its object is to restate some of the results of the first chapter in a form better suited for the applications in the following sections.

If $A$ and $B$ are any two points in the affine plane, then there exists one and only one translation of the affine plane, mapping $A$ upon $B$ (I.3, Theorem 3 , and I.4, Corollary 2). In accordance with customary terminology this translation may be termed $a$ vector and the ordered pair $|A B|$ may be said to represent this vector. In particular $|A B|$ and $\left|A^{\prime} B^{\prime}\right|$ represent the same vector, in symbols $|A B| \sim\left|A^{\prime} B^{\prime}\right|$, if, and only if, $A$ is mapped upon $B$ by the same translation that maps $A^{\prime}$ upon $B^{\prime}$. We enumerate some fundamental properties of vector equality.

V.1. Vector equality is reflexive, symmetric and transitive.

V.2. $|A A| \sim\left|A^{\prime} B^{\prime}\right|$ if, and only if, $A^{\prime}=B^{\prime}$.

V.3. Suppose that $A \neq B, A^{\prime} \neq B^{\prime}$ and $A+B \neq A^{\prime}+B^{\prime}$. Then $|A B|$ $\sim\left|A^{\prime} B^{\prime}\right|$ if, and only if, $A+B \| A^{\prime}+B^{\prime}$ and $A+A^{\prime} \| B+B^{\prime}$.

V.4. Suppose that $A \neq B, A^{\prime} \neq B^{\prime}$ and that $A+B=A^{\prime}+B^{\prime}$. Then $|A B|$ $\sim\left|A^{\prime} B^{\prime}\right|$ if, and only if, there exist two different points $A^{\prime \prime}$ and $B^{\prime \prime}$ not on $A+B$ such that $A^{\prime \prime}+B^{\prime \prime}\left\|A+B, A+A^{\prime \prime}\right\| B+B^{\prime \prime}, A^{\prime}+A^{\prime \prime} \| B^{\prime}+B^{\prime \prime}$.

V.5. $|A B| \sim\left|A^{\prime} B^{\prime}\right|$ if, and only if, $\left|A A^{\prime}\right| \sim\left|B B^{\prime}\right|$.

V.6. $B=A \circ C$ if, and only if, $A, B, C$ are three different collinear points satisfying $|A B| \sim|B C|$.

The properties V.1 to V.4 are immediate consequences of the definition of vector equality and of the fact, already mentioned, that the group of translations is simply transitive. V.5 is readily deduced from V.3; and V.6 is a consequence of V.4 because of I.2, Theorem $4(\mathrm{v})$.

We mention finally that it would be possible to deduce the simple transitivity of the group of translations from V.1 and V.3. 
III.2. The congruence relation induced by an orthogonality relation. The following lemma will prove useful later on.

LEMMA 1. If the orthogonality relation $R$ meets the requirements 0.1 to 0.3 , if the three points $A, B, C$ are not collinear, and if $A^{\prime}=B \circ C, B^{\prime}=C \circ A$, $C^{\prime}=A \circ B$, then each of the following properties implies all the others.

(i) $A^{\prime}+B^{\prime} \perp A+C$.

(ii) $A+C \perp A+B$.

(iii) $A+B \perp A^{\prime}+C^{\prime}$.

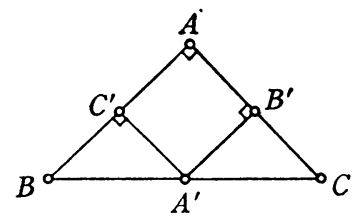

FIG. 10

Proof. It is clear that it suffices to prove the equivalence of (i) and (ii); and this equivalence is an immediate consequence of the parallelism of the lines $A+B$ and $A^{\prime}+B^{\prime}$ which in its turn may be deduced from I.5, Theorem 1 .

If the two pairs of points $A B$ and $A^{\prime} B^{\prime}$ are congruent under some congruence relation $Q$, then we shall express this fact in the usual way by $A B \equiv A^{\prime} B^{\prime}$. If there is a possibility of confusion, then we shall say that these two pairs of points are $Q$-congruent.

We shall now connect with every orthogonality relation $R$ which meets the requirements 0.1 to 0.3 a congruence relation $K(R)$ by the following two rules.

(a) If the pairs of points $A, B$ and $A^{\prime}, B^{\prime}$ are situated on parallel lines, then $A B \equiv A^{\prime} B^{\prime}$ is a necessary and sufficient condition for the validity of one of the relations $|A B| \sim\left|A^{\prime} B^{\prime}\right|$ and $|A B| \sim\left|B^{\prime} A^{\prime}\right|$.

(b) If the pairs of points $A, B$ and $A^{\prime}, B^{\prime}$ are not situated on parallel lines, then $A B \equiv A^{\prime} B^{\prime}$ is a necessary and sufficient condition for the existence of a point $H$ such that

$$
|A B| \sim\left|A^{\prime} H\right| \text { and } \quad H+B^{\prime} \perp A^{\prime}+\left(B^{\prime} \circ H\right) .
$$

(See Fig. 11 below.)

REMARKS. 1. It is readily verified that $K(R)$ is uniquely determined by $R$.

2. If $A=B$, then there exist parallel lines containing $A, B$ and $A^{\prime}, B^{\prime}$ respectively. Thus condition (b) will not arise unless $A \neq B, A^{\prime} \neq B^{\prime}$, in which case $|A B| \sim\left|A^{\prime} H\right|$ implies that $H$ is not on $A^{\prime}+B^{\prime}$.

3. If $A=A^{\prime}$, and if (b) applies, then we deduce from $|A B| \sim\left|A^{\prime} H\right|$ that $H=B$. Thus we obtain the following important special case of condition (b). 
(b') If the three points $A, B, C$ are not collinear, then $A B \equiv B C$ is a necessary and sufficient condition for $A+C \perp B+(A \circ C)$.

It is clear that (b) may be deduced from (a), ( $\left.b^{\prime}\right)$ and the postulate that congruence be transitive. But we cannot make such a postulate at this stage of the development, as follows from Theorem 2 below.

THEOREM 1. If postulates 0.1 to 0.3 are satisfied by the orthogonatity relation $R$, then the congruence relation $K(R)$ has the following properties.

(1) $A B \equiv A^{\prime} B^{\prime}$ if (and only if) $B A \equiv A^{\prime} B^{\prime}$.

(2) $A B \equiv A^{\prime} B^{\prime}$ if (and only if) $A^{\prime} B^{\prime} \equiv A B$.

Proof. Both our contentions are obvious in case the pairs $A, B$ and $A^{\prime}, B^{\prime}$ are situated on parallel lines. Thus we assume now that this be not the case. If $A B \equiv A^{\prime} B^{\prime}$, then there exists by (b) a point $H$ such that $|A B| \sim\left|A^{\prime} H\right|$ and $H+B^{\prime} \perp A^{\prime}+\left(B^{\prime} \circ H\right)$. From the properties of the midpoint relation (I.2, IV) we infer the existence of one and only one point $L$ such that

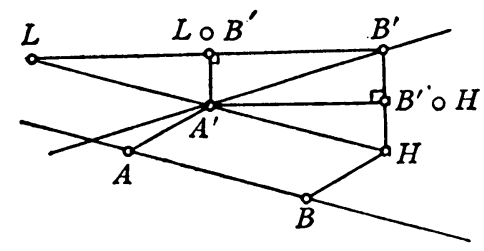

FIG. 11

$A^{\prime}=L \circ H$. It is a consequence of III.11, V.1, and V.6 that $|A B| \sim\left|L A^{\prime}\right|$ and that therefore $|B A| \sim\left|A^{\prime} L\right|$; and applying Lemma 1, (i), (iii) on the triangle $B^{\prime}, H, L$ we infer $L+B^{\prime} \perp A^{\prime}+\left(L \circ B^{\prime}\right)$, proving (1).

To prove (2) we consider the uniquely determined translation $t$ which maps $A^{\prime}$ upon $A$. This translation maps $H$ upon $B$ and $B^{\prime}$ upon some point $M$. It maps furthermore $B^{\prime} \circ H$ upon $M \circ B$ and it maps pairs of perpendicular lines upon pairs of perpendicular lines, since translations map every line upon a parallel line. Thus we infer $\left|A^{\prime} B^{\prime}\right| \sim|A M|$ from III.1, V.5 and we have $M+B \perp A+(M \circ B)$, proving (2).

THEOREM 2. Suppose that postulates 0.1 to 0.3 are satisfied by the orthogonality relation $R$ and that not every line is $R$-self-perpendicular. Then the congruence relation $K(R)$ is transitive if, and only if, 0.6 is satisfied by $R$.

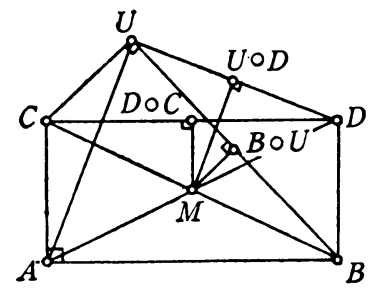

Fig. 12 
Proof. Suppose first that $K(R)$ is transitive; and assume that no three of the four points $A, B, C, D$ are collinear, that $A+B\|C+D, A+C\| B+D$, $A+B \perp A+C$, and that $U$ is a point different from $A, B, C, D$ satisfying $B+U \perp C+U$. Then the lines $A+D$ and $B+C$ are not parallel( $\left.{ }^{15}\right)$ and they meet in the midpoint $M$ of both $A D$ and $B C$. Thus it follows from part (a) of the definition of $K(R)$ that $A M \equiv M D$ and $B M \equiv M C$. It is a consequence of Lemma 1 that $D+C \perp M+(D \circ C)$. Thus it follows from condition (b) (or $\left(\mathrm{b}^{\prime}\right)$ ) of the definition of $K(R)$ that $C M \equiv M D$; and we deduce from the transitivity of congruence that $A M, B M, C M$ and $D M$ are congruent. It is a consequence of Lemma 1 and our hypotheses that $B+U$ is perpendicular to $M+(B \circ U)$; and thus we infer from condition (b) (or ( $\left.\mathrm{b}^{\prime}\right)$ ) that $B M \equiv M U$. Hence $M U$ and $M D$ are congruent because of the transitivity of congruence; and we deduce from $\left(\mathrm{b}^{\prime}\right)$ that $U+D$ and $M+(U \circ D)$ are perpendicular. Hence it follows from Lemma 1 that $A+U$ and $U+D$ are perpendicular, proving that 0.6 is satisfied by $R$.

Suppose conversely that 0.6 is satisfied by the orthogonality relation $R$. Then we deduce from II.1, Theorem 2, that the affine plane under consideration is the plane over an ordinary commutative field $F$ of characteristic different from 2, that there exists a rectangular system of $x=y$-coordinates, and that the lines $y=x a+b$ and $y=x a^{*}+d$ for $a \neq 0$ and $a^{*} \neq 0$ are perpendicular if, and only if, $a a^{*}=c$ where $c$ is the constant of orthogonality of the rectangular system of coordinates which we are using. We prove next the following lemma.

(2.1) If $(r, s),\left(r^{\prime}, s^{\prime}\right),(u, v),\left(u^{\prime}, v^{\prime}\right)$ are four points in the plane, then the following equation is a necessary and sufficient condition for $K(R)$-congruence of $(r, s)(u, v)$ and $\left(r^{\prime}, s^{\prime}\right)\left(u^{\prime}, v^{\prime}\right)$.

$$
(v-s)^{2}-c(u-r)^{2}=\left(v^{\prime}-s^{\prime}\right)^{2}-c\left(u^{\prime}-r^{\prime}\right)^{2} .
$$

Since every translation $t$ may be described in the form $(x, y)^{t}=(x+a, y+b)$ for suitable numbers $a, b$ in $F$, it follows that the form $\left(y-y^{\prime}\right)^{2}-c\left(x-x^{\prime}\right)^{2}$ is invariant under translations. Thus we may assume without loss of generality that $(r, s)=\left(r^{\prime}, s^{\prime}\right)$, since the group of translations is simply transitive. One verifies as usual that $(u, v) \circ\left(u^{\prime}, v^{\prime}\right)=\left(\left(u+u^{\prime}\right) / 2,\left(v+v^{\prime}\right) / 2\right)$ and that $(r, s)+\left(\left(u+u^{\prime}\right) / 2,\left(v+v^{\prime}\right) / 2\right)$ is perpendicular to $(u, v)+\left(u^{\prime}, v^{\prime}\right)$ if, and only if, $\left(\left(v+v^{\prime}\right) / 2-s\right)\left(v^{\prime}-v\right)=c\left(\left(u+u^{\prime}\right) / 2-r\right)\left(u-u^{\prime}\right)$. By direct computation we find that the last condition is equivalent to $(v-s)^{2}-c(u-r)^{2}=\left(v^{\prime}-s\right)^{2}$ $-c\left(u^{\prime}-r\right)^{2}$; and this completes the proof of Lemma 2.1 .

The transitivity of $K(R)$-congruence is an immediate consequence of (2.1), and this completes the proof of our theorem.

REMARK. In connection with Lemma $2.1 \mathrm{cp}$. the results of §II.4. Note furthermore the impossibility of simplifying the second part of the proof of

(15) This is a consequence of Fano's axiom; see I.2, Remark to Theorem 3. 
the theorem by reference to the group of rotations, since the existence of sufficiently many rotations (or of congruence preserving affinities) has not yet been assured. See in this respect below III.3, Corollary 2.

III.3. The orthogonality relation induced by a congruence relation. If $Q$ is a congruence relation between pairs of points, then the derived orthogonality relation $P(Q)$ is defined by the following rule.

(c) The line $r$ is $P(Q)$-perpendicular to the line $s$ if, and only if, there exist points $R \neq R^{\prime}$ and $S \neq S^{\prime}$ satisfying

$$
r=R+R^{\prime}, \quad s=S+S^{\prime}, \quad S R \equiv R S^{\prime}, \quad S R^{\prime} \equiv R^{\prime} S^{\prime} .
$$

It should be noted that this definition of perpendicularity is not symmetric in the lines $r$ and $s$. other.

THEOREM. The following properties of the congruence relation $Q$ imply each

(A) $Q=K(P(Q))$ and 0.1 to 0.4 are satisfied by the orthogonality relation $P(Q)$ without self-perpendicular lines.

(B) There exists an orthogonality relation $R$ without self-perpendicular lines satisfying 0.1 to 0.4 and $Q=K(R)$.

(C) The following conditions are satisfied by $Q$.

C.1. $A B \equiv A^{\prime} B^{\prime}$ if (and only if) $B A \equiv A^{\prime} B^{\prime}$.

C.2. $Q$ is symmetric, reflexive and transitive.

C.3. If the pairs of points $A, B$ and $A^{\prime}, B^{\prime}$ are situated on parallel lines, then $A B \equiv A^{\prime} B^{\prime}$ is a necessary and sufficient condition for the validity of one of the relations $|A B| \sim\left|A^{\prime} B^{\prime}\right|$ and $|A B| \sim\left|B^{\prime} A^{\prime}\right|$.

C.4. The locus of the points $X$ satisfying $U X \equiv X V$ for given $U \neq V$ is a line not $U+V$.

Proof. It is obvious that (B) is a consequence of (A). Thus we assume now that (B) be satisfied by $Q$. Then we deduce C.1 and C.2 from II.1, Theorem 2, and III.2, Theorems 1 and 2. Condition C.3 is nothing but a restatement of III.2 (a) which condition is part of the definition of $K(R)$. If $U$ and $V$ are two different points, then there exists one and only one line $w$ which is $R$-perpendicular to $U+V$ and which passes through the midpoint $U \circ V$. It is an immediate consequence of III.2 (b) (part of the definition of $K(R)$ ) that the point $X$ is on the line $w$ if, and only if, $U X \equiv X V$, proving the validity of C.4, since $w \neq U+V$.

We assume finally that the postulates C. 1 to C. 4 are satisfied by the congruence relation $Q$. If $U$ and $V$ are two different points, then it follows from C.4 that the locus of all the points $X$ satisfying $U X \equiv X V$ is a well determined line $m(U, V) \neq U+V$. We deduce $m(U, V)=m(V, U)$ from C.1 and C.2 ; and it is a consequence of C.3 that the midpoint $U \circ V$ is on $m(U, V)$. We prove:

(1.1) If $Y \neq Y^{\prime}$ are points on $m(U, V)$ which satisfy $Y \circ Y^{\prime}=U \circ V$, then $m\left(Y, Y^{\prime}\right)=U+V$. 
Since the reflection in the point $W=U \circ V=Y \circ Y^{\prime}$ interchanges $U$ and $V$ as well as $Y$ and $Y^{\prime}$ (by I.3, Theorem 1), and since a reflection maps every line upon a parallel one, we deduce that $U+Y \| V+Y^{\prime}$ and $U+Y^{\prime} \| V+Y$. Thus $U Y \equiv V Y^{\prime}$ and $U Y^{\prime} \equiv V Y$ are consequences of C.3 and III.1, V.3. Consequently $U$ and $V$ belong to $m\left(Y, Y^{\prime}\right)$. But $m\left(Y, Y^{\prime}\right)$ has been shown to be a line. Hence $U+V=m\left(Y, Y^{\prime}\right)$.

(1.2) If $U, U^{\prime}$ and $V, V^{\prime}$ are two pairs of different points such that $U+U^{\prime}$ $=V+V^{\prime}$ and $U \circ U^{\prime}=V \circ V^{\prime}$, then $m\left(U, U^{\prime}\right)=m\left(V, V^{\prime}\right)$.

There exists a pair of different points $Y, Y^{\prime}$ on $m\left(U, U^{\prime}\right)$ such that $Y \circ Y^{\prime}=U \circ U^{\prime}$. Then it follows from (1.1) that $m\left(Y, Y^{\prime}\right)=U+U^{\prime}=V+V^{\prime}$. Hence $V, V^{\prime}$ is a pair of different points on $m\left(Y, Y^{\prime}\right)$, satisfying $Y \circ Y^{\prime}$ $=V \circ V^{\prime}$; and it follows from (1.1) that $m\left(V, V^{\prime}\right)=Y+Y^{\prime}$. But $Y+Y^{\prime}$ $=m\left(U, U^{\prime}\right)$ by our selection of the points $Y, Y^{\prime}$, proving $m\left(U, U^{\prime}\right)=m\left(V, V^{\prime}\right)$.

If $Z$ is a point on the line $z$, then we define $m(Z, z)=m\left(U, U^{\prime}\right)$ where $U, U^{\prime}$ is any pair of different points satisfying $Z=U \circ U^{\prime}$ and $z=U+U^{\prime}$. It is a consequence of (1.2) that the line $m(Z, z)$ is independent of the special choice of the pair $U, U^{\prime}$. We note furthermore that $m(Z, z)$ is different from $z$ and passes through $Z$.

(1.3) $z=m(Z, m(Z, z))$ for $Z$ on $z$.

This is an almost immediate consequence of (1.1).

(1.4) $m(Z, z) \| m\left(Z^{\prime}, z\right)$ for $Z$ and $Z^{\prime}$ on $z$.

There exists one and only one translation $t$ mapping $Z$ upon $Z^{\prime}$. It is a consequence of C.3 and III.1, V.3, that $A B \equiv A^{t} B^{t}$ for points $A$ and $B$. Hence $m(Z, z)^{t}=m\left(Z^{\prime}, z\right)$, since $t$ maps $z$ into itself. But translations map every line upon a parallel one, proving our contention.

Applying condition (c) and the facts already established, it follows now that $m(Z, z)$ is uniquely determined as the line which is $P(Q)$-perpendicular to $z$ and passes through $Z$. From (1.3) and (1.4) we deduce that 0.1 to 0.3 are satisfied by $P(Q)$; and there do not exist $P(Q)$-self-perpendicular lines, since $m(Z, z) \neq z$. From C. 3 and C.4 it is readily deduced that $Q=K(P(Q))$. Hence it follows from III.2, Theorem 2 , that 0.6 is satisfied by $P(Q)$; and we infer from II.1, Theorem 2 , that 0.4 is satisfied by $P(Q)$. Thus (A) has been shown to be a consequence of $(C)$, completing the proof of the theorem.

COROLlARY 1. If 0.1 to 0.4 are satisfied by the orthogonality relation $R$ without self-perpendicular lines, then $R=P(K(R))$.

Proof. We infer from the Theorem that C. 1 to C.4 are satisfied by $K(R)$; and thus it follows from the Theorem that 0.1 to 0.4 are satisfied by $P(K(R))$ and that no lines are $P(K(R))$-self-perpendicular. If the lines $r$ and $s$ are $R$-perpendicular, then consider any point $X$ on $r$ not on $s$. There exists one and only one point $X^{\prime}$ on $r$ such that $X \circ X^{\prime}=r s$. It is an immediate consequence of the definition of $K(R)$ (see III.2, ( $\left.\mathrm{b}^{\prime}\right)$ !) that a point $Y$ is on $s$ if, and only if, $X Y \equiv Y X^{\prime}$. But then we deduce from the definition of $F(K(R))$ that $s$ is $P(K(R))$-perpendicular to $s$ too. Since there exists through any given 
point $Z$ one and only one line which is $R$-perpendicular to a given line $z$ and one and only one line which is $P(K(R))$-perpendicular to $z$, we deduce now the identity of $R$ and $P(K(R))$.

An affinity of the plane is a $1: 1$ correspondence $a$ of the points of the plane which preserves collinearity. If $Q$ is a congruence relation, then the affinity $a$ is termed a $Q$-affinity, if $A B \equiv A^{a} B^{a}$ for any two points $A$ and $B$ of the plane.

CoROllaRY 2: If C.1 to C.4 are satisfied by the congruence relation $Q$, and if $A, B, A^{\prime}, B^{\prime}$ are points, then $A B \equiv A^{\prime} B^{\prime}$ is a necessary and sufficient condition for the existence of a $Q$-affinity a satisfying $A^{\prime}=A^{a}$ and $B^{\prime}=B^{a}$.

Proof. Suppose that $A B \equiv A^{\prime} B^{\prime}$. Since translations are $Q$-affinities, we may assume without loss of generality that $A=A^{\prime}$ and that the points $A, B, B^{\prime}$ are not collinear. It is now readily deduced from the theorem and from II.1, Theorem 2, that there exists a $P(Q)$-rectangular system of coordinates (with values in an ordinary commutative field of characteristic different from 2) such that $A+\left(B \circ B^{\prime}\right)$ is its $x$-axis. Then the transformation $x^{\prime}=x$, $y^{\prime}=-y$ is a $Q$-affinity interchanging $B$ and $B^{\prime}$ and having $A$ for a fixed point, since this transformation preserves $P(Q)$-perpendicularity and since $Q$ $=K(P(Q))$.

Applying both Corollary 2 and the arguments of its proof one readily verifies the following theorem.

CoRollary $2^{\prime}$. If $\mathrm{C} .1$ to $\mathrm{C} .4$ are satisfied by the congruence relation $Q$, if $A, A^{\prime}, B, B^{\prime}, C, C^{\prime}$ are points satisfying $A B \equiv A^{\prime} B^{\prime}, B C \equiv B^{\prime} C^{\prime}$ and $C A \equiv C^{\prime} A^{\prime}$, then there exists a Q-affinity mapping $A$ upon $A^{\prime}, B$ upon $B^{\prime}, C$ upon $C^{\prime}$.

Using III.2, Lemma 1 , and the considerations of $\$ I I .4$ one verifies readily that the $Q$-affinities with fixed point $A$ are just those affinities with fixed point $A$ which preserve $P(Q)$-perpendicularity and the circles around $A$ which are defined on the basis of $P(Q)$, provided that C.1 to C.4 are satisfied by $Q$.

There arises finally the question whether it may be possible to characterize the congruence relation $Q$ by means of the orthogonality relation $P(Q)$. That this is impossible may be seen from the following example of an "incomplete" congruence relation.

Denote by $F$ some real field in which sums of squares are squares and consider the affine plane over $F$. Define congruence in this affine plane by means of the following two rules.

(i) If the two pairs of points $A, B$ and $A^{\prime}, B^{\prime}$ are situated on parallel lines, then $A B \equiv A^{\prime} B^{\prime}$ is a necessary and sufficient condition for the validity of at least one of the relations: $|A B| \sim\left|A^{\prime} B^{\prime}\right|$ and $|A B| \sim\left|B^{\prime} A^{\prime}\right|$.

(ii) If the two pairs of points $(r, s),(u, v)$ and $\left(r^{\prime}, s^{\prime}\right),\left(u^{\prime}, v^{\prime}\right)$ are not situated on parallel lines, then $A B \equiv A^{\prime} B^{\prime}$ is a necessary and sufficient condition for

$$
(r-u)^{2}+(s-v)^{2}=\left(r^{\prime}-u^{\prime}\right)^{2}+\left(s^{\prime}-v^{\prime}\right)^{2}=1 .
$$


It is readily seen that C. 1 to C.3, but not C.4, are satisfied by this congruence relation $Q$; and that the orthogonality relation $P(Q)$ is just the ordinary perpendicularity, defined by considering the present system of coordinates as rectangular coordinates and using the constant of orthogonality $c=-1$.

E. ArTin

\section{BiBLIOGRAPHY}

1. Coordinates in affine geometry, Reports of a mathematical colloquium, University of Notre Dame, (2) vol. 2 (1940) pp. 15-20.

F. BachmanN

1. Eine Begründung der absoluten Geometrie, Math. Ann. vol. 113 (1937) pp. 424-451.

2. Stufen der absoluten Geometrie, die Frage nach der Unabhängigkeit der Anordnung, Math. Ann. vol. 117 (1940) pp. 197-234.

R. BAER

1. Homogeneity of projective planes, Amer. J. Math. vol. 64 (1942) pp. 137-152.

R. BALDUS

1. Über Euler's Dreieckssatz in der absoluten Geometrie, Heidelberg Akad. Wiss. Sitzungsber. (1929) p. 11.

O. Botrema

1. Zur Axiomatik der projektiven Geometrie, Monatshefte für Mathematik und Physik vol. 47 (1937) pp. 93-121.

2. De elementaire meetkunde van het platte vlak, Groningen, Batavia, 1938.

3. Eine Geometrie mit unvollständiger Anordnung, Math. Ann. vol. 117 (1940) pp. 17-26.

D. HilberT

1. Grundlagen der Geometrie, 7th ed. Leipzig and Berlin, 1930.

J. HJELMSLEV

1. Neue Begrïndung der ebenen Geometrie, Math. Ann. vol. 64 (1907) pp. 449-474.

2. Einleitung in die allgemeine Kongruenzlehre, Danske Videnskabernes Selskab-Mathematisk-fysiske meddelelser vol. 8 (1929) and vol. 10 (1929).

E. KAMKE

1. Zur Definition der affinen Abbildung. Jber. Deutschen Math. Verein. vol. 36 (1927) pp. $145-156$.

J. MOLLERUP

1. Studier over den plane geometris Aksiomer, Kjobnhavn, 1903.

R. Moufang

1. Alternativkörper und der Satz vom vollständigen Vierseit $\left(D_{\bullet}\right)$, Abh. Math. Hamburgischen Univ vol. 9 (1933) pp. 207-222.

K. REIDEMEISTER

1. Grundlagen der Geometrie, Berlin, 1930.

F. SCHUR

1. Zur Proportionenlehre, Math. Ann. vol. 57 (1903) pp. 205-208.

2. Grundlagen der Geometrie, Leipzig and Berlin, 1909.

G. THOMSEN

1. Grundlagen der Elementargeometrie in gruppentheoretischer Behandlung, Leipzig and Berlin, 1933.

H. TOEPKEN

1. Über den Höhensatz in der absoluten Geometrie, Deutsche Mathematik vol. 5 (1941) pp. 395-401.

UNIVERSITY OF ILLINOIS,

URBANA, Ill. 